TRANSACTIONS OF THE

AMERICAN MATHEMATICAL SOCIETY

Volume 361, Number 1, January 2009, Pages 343-390

S 0002-9947(08)04668-0

Article electronically published on August 21, 2008

\title{
NECESSARY AND SUFFICIENT CONDITIONS FOR VIABILITY FOR SEMILINEAR DIFFERENTIAL INCLUSIONS
}

\author{
OVIDIU CÂRJĂ, MIHAI NECULA, AND IOAN I. VRABIE
}

\begin{abstract}
Given a set $K$ in a Banach space $X$, we define: the tangent set, and the quasi-tangent set to $K$ at $\xi \in K$, concepts more general than the one of tangent vector introduced by Bouligand (1930) and Severi (1931). Both notions prove very suitable in the study of viability problems referring to differential inclusions. Namely, we establish several new necessary, and even necessary and sufficient conditions for viability referring to both differential inclusions and semilinear evolution inclusions, conditions expressed in terms of the tangency concepts introduced.
\end{abstract}

\section{INTRODUCTION}

The aim of this paper is to prove some necessary and also some necessary and sufficient conditions in order that a given subset $K$ of a real Banach space $X$ be viable with respect to a multi-function $F: K \leadsto X$, i.e., a function $F: K \rightarrow 2^{X}$, or with respect to $A+F, A: D(A) \subseteq X \rightarrow X$ being the infinitesimal generator of a $C_{0}$-semigroup, $\{S(t): X \rightarrow X ; t \geq 0\}$, on $X$. More precisely, we consider the differential inclusions

$$
u^{\prime}(t) \in F(u(t))
$$

and

$$
u^{\prime}(t) \in A u(t)+F(u(t))
$$

and we are interested in finding necessary and even necessary and sufficient conditions in order that for each $\xi \in K$, at least one solution $u:[0, T] \rightarrow K$ of (1.1) or (1.2) exists satisfying the initial condition $u(0)=\xi$. Of course, the notion of solution for both (1.1) and (1.2) must be defined properly and we will do that later on.

As far as (1.1) is concerned, the viability problem has been studied by many authors by using various frameworks and techniques. We begin by noticing the pioneering work of Nagumo [18] who considered the case $X$ finite dimensional and $F$ single-valued and continuous. In the case when $X$ is finite dimensional but $F$ is multi-valued, the first necessary and sufficient condition for viability, extending Nagumo's main result in [18] from ordinary differential equations to differential

Received by the editors February 15, 2007.

2000 Mathematics Subject Classification. Primary 34G20, 47J35; Secondary 35K57, 35K65.

Key words and phrases. Viability, tangency condition, reaction-diffusion systems, compact semigroup.

The first and third authors were supported by the Project CEx05-DE11-36/05.10.2005. The second author was supported by CNCSIS Grant A 1159/2006.

(C)2008 American Mathematical Society Reverts to public domain 28 years from publication 
inclusions, was given by Bebernes-Schuur 2]. More precisely, Bebernes-Schuur 2 2 showed that, whenever $F$ is upper semi-continuous (u.s.c.) with nonempty, convex, closed and bounded values and $K$ is locally closed, then $K$ is viable with respect to $F$ if and only if

$$
F(\xi) \cap \mathcal{T}_{K}(\xi) \neq \emptyset
$$

for each $\xi \in K$, where $\mathcal{T}_{K}(\xi)$ denotes the contingent cone to $K$ at $\xi \in K$. We recall that $\mathcal{T}_{K}(\xi)$ consists of all vectors $\eta \in X$ which satisfy

$$
\liminf _{h \downarrow 0} \frac{1}{h} \operatorname{dist}(\xi+h \eta ; K)=0 .
$$

We also recall that $K$ is locally closed if for each $\xi \in K$ there exists $\rho>0$ such that $K \cap D(\xi, \rho)$ is closed, $D(\xi, \rho)$ being the closed ball with center $\xi$ and radius $\rho$.

If $X$ is infinite dimensional, one may easily obtain an extension of this result by assuming that $K$ is locally compact and $F$ has nonempty, convex and compact values. See, for example, Aubin-Cellina [1, Proposition 1 and Theorem 1, p. 180. Clarke-Ledyaev-Radulescu [9] consider the case of an infinite-dimensional Hilbert space $X$ and relax the assumption on $K$ from locally compact to locally closed. Instead, they strength the assumption on $F$, by assuming that

$(C H)$ there exists $k>0$ such that for any bounded set $D \subseteq X$,

$$
\alpha(F(D)) \leq k \alpha(D)
$$

where $\alpha$ denotes the Kuratowski measure of noncompactness. Under these circumstances, Clarke-Ledyaev-Radulescu 9 proved that $K$ is viable with respect to $F$ if and only if (1.3) holds true for each $\xi \in K$. Of course, the condition $(C H)$ implies the compactness of the values of $F$. Assuming that the multi-function $F$ has closed and bounded (instead of compact) values and that $K$ is locally compact, Cârjă and Monteiro Marques [6] obtain a necessary and sufficient condition for the viability of $K$ with respect to $F$ of the type (1.3) but with a weak tangency concept in (1.4).

At this point, we have to mention the work of Gautier [15] who assumes that $K$ is weakly closed and $F$ is weakly-weakly u.s.c. with closed convex and bounded values and obtains a sufficient condition for viability via a weak tangency condition.

Here we introduce a new tangency concept (involving strong convergence) that allows us to get a tangency condition that is necessary and sufficient for the viability of a locally closed set $K$ with respect to a nonempty, closed, convex with bounded values, u.s.c. multi-function. More precisely, we say that a set $E \subseteq X$ is tangent to $K$ at $\xi \in K$ if

$$
\liminf _{h \downarrow 0} \frac{1}{h} \operatorname{dist}(\xi+h E ; K)=0 .
$$

Now, the corresponding tangency condition for viability is

(TC1) for each $\xi \in K$ the set $F(\xi)$ is tangent to $K$ at $\xi$.

Clearly (TC1) reduces to (1.3) whenever $F$ has compact values.

Concerning the semilinear differential inclusion (1.2), i.e. $A$ unbounded and $F$ multi-valued, as far as we know, it was first considered by Pavel-Vrabie [19], 20]. For subsequent developments, see Shi [22], Cârjă-Vrabie [8] and the references therein. We recall that Pavel-Vrabie [19, 20] assume that $X$ is reflexive, $K$ is locally closed, $F$ is a locally bounded and nonempty, closed and convex valued multi-function with strongly-weakly sequentially closed graph, while $A$ generates 
a compact $C_{0}$-semigroup. Within this general setting, they prove that if for each $\xi \in K$ and each $y \in F(\xi)$,

$$
\lim _{h \downarrow 0} \frac{1}{h} \operatorname{dist}(S(h) \xi+h y ; K)=0,
$$

then $K$ is viable with respect to $A+F$. Shi 22 considers the case in which $K$ is compact, $F$ is u.s.c and nonempty, convex and compact valued, and $A$ generates a compact and differentiable $C_{0}$-semigroup. Under these assumptions, Shi 22 proves that $K$ is viable with respect to $A+F$ if and only if for each $\xi \in K$ there exists $y \in F(\xi)$ such that $y \in \mathcal{T}_{K}^{A}(\xi)$, i.e.,

$$
\liminf _{h \downarrow 0} \frac{1}{h} \operatorname{dist}(S(h) \xi+h y ; K)=0 .
$$

Clearly, if $A \neq 0$, the tangency condition (1.7) is significantly weaker than (1.6) introduced in Pavel-Vrabie [19], 20, but it is quite close to (1.6) when $A=0$. In addition, it is necessary and sufficient for viability. Nevertheless, the general assumptions imposed by Shi 22] on $F$ and $K$ are stronger than the ones in PavelVrabie [19], 20]. For instance, if $X$ is an infinite-dimensional function space and $F$ is a superposition operator which is not single-valued, the compactness of $F(\xi)$ is ruled out. More than this, the compactness assumption on the values of $F$ is also too strong when dealing with differential inclusions of the form (1.4) coming from optimal control problems. In order to relax this hypothesis, Cârjă-Vrabie [8] assume that $A$ generates a $C_{0}$-semigroup, $K$ is weakly locally closed and $F$ is weakly-weakly u.s.c. with nonempty, closed, convex and bounded values. Then, they prove a necessary and sufficient condition in order that $K$ be viable with respect to $A+F$ expressed in the terms of a weak tangency concept. However, the price payed for this relaxation, i.e., the assumption that $F$ is weakly-weakly u.s.c., is rather high.

Therefore, in order to get necessary as well as necessary and sufficient conditions for viability of a set $K$ with respect to $A+F$ as suitable for applications as possible, in this paper, we introduce two new tangency concepts which lead to two new tangency conditions reducing to (1.7) when $F$ is compact-valued. Both are intended to handle not only the already known cases but also the ones which are not covered by the general viability results mentioned above. The first new tangency condition extends (1.7) by simply putting $F(\xi)$ instead of $y$. Namely, we assume that (TC2) for each $\xi \in K$,

$$
\liminf _{h \downarrow 0} \frac{1}{h} \operatorname{dist}(S(h) \xi+h F(\xi) ; K)=0 .
$$

The second one reads as follows:

(TC3) For each $\xi \in K$,

$$
\liminf _{h \downarrow 0} \frac{1}{h} \operatorname{dist}\left(S_{\mathcal{F}(\xi)}(h) \xi ; K\right)=0,
$$

where

$$
\mathcal{F}(\xi)=\left\{f \in L^{1}\left(\mathbb{R}_{+} ; X\right) ; f(s) \in F(\xi) \text { a.e. for } s \in \mathbb{R}_{+}\right\}
$$

and

$$
S_{\mathcal{F}(\xi)}(h) \xi=\left\{S(h) \xi+\int_{0}^{h} S(h-s) f(s) d s ; f \in \mathcal{F}(\xi)\right\} .
$$


We have to notice that both $(T C 2)$ and $(T C 3)$ reduce to $(T C 1)$ when $A=0$. We also emphasize that the tangency condition $(T C 3)$ is necessary and sufficient for viability without assuming the compactness of $F(\xi)$ and is suitable in establishing the viability of an epigraph, that, in its turn, leads to a controllability result. See the application in the last Section 18.

The outline of the paper is as follows. In Section 2 we collect some notation, basic definitions and preliminary results in Functional Analysis. In Section 3 we introduce the notion of a tangent set, we recall the definition of tangent vector and clarify the relationship between them. Sections $4 \sim 7$ are devoted to the proof of necessary and also necessary and sufficient conditions for the viability of $K$ with respect to $F$. The nonautonomous case is considered in Section 8 while the existence of global solutions of (1.1) is studied in Section 9. In Section 10 we introduce the new concepts of $A$-tangent set and $A$-quasi-tangent set to $K$ at $\xi \in K$, which are very appropriate for studying the viability of $K$ with respect to $A+F$. Necessary and even necessary and sufficient conditions for this kind of viability are given in Sections $11 \sim 15$. The quasi-autonomous case of $(1.2)$ is considered in Section 16, while the existence of a global solution is studied in Section 17. Finally, in Section 18, we give an interesting application of one of our viability results in obtaining a sufficient condition of null controllability for a semilinear evolution equation.

\section{Preliminaries}

2.1. Brezis-Browder ordering principle. To begin with, let us recall some definitions and notation. Let $\mathcal{S}$ be a nonempty set. A binary relation $\preceq \subseteq \mathcal{S} \times \mathcal{S}$ is a preorder on $\mathcal{S}$ if it is reflexive, i.e., $\xi \preceq \xi$ for each $\xi \in \mathcal{S}$, and transitive, i.e., $\xi \preceq \eta$ and $\eta \preceq \zeta$ imply $\xi \preceq \zeta$.

Definition 2.1. Let $\mathcal{S}$ be a nonempty set, $\preceq \subseteq \mathcal{S} \times \mathcal{S}$ a preorder on $\mathcal{S}$, and let $\mathcal{N}: \mathcal{S} \rightarrow \mathbb{R} \cup\{+\infty\}$ be an increasing function. An $\mathcal{N}$-maximal element is an element $\bar{\xi} \in \mathcal{S}$ satisfying $\mathcal{N}(\xi)=\mathcal{N}(\bar{\xi})$, for every $\xi \in \mathcal{S}$ with $\bar{\xi} \preceq \xi$.

We may now proceed to the statement of the main result in this section, i.e., the Brezis-Browder ordering principle :

Theorem 2.1. Let $\mathcal{S}$ be a nonempty set, $\preceq \subseteq \mathcal{S} \times \mathcal{S}$ a preorder on $\mathcal{S}$ and let $\mathcal{N}: \mathcal{S} \rightarrow \mathbb{R} \cup\{+\infty\}$ be a given function. Suppose that:

(i) each increasing sequence in $\mathcal{S}$ is bounded from above;

(ii) the function $\mathcal{N}$ is increasing.

Then, for each $\xi_{0} \in \mathcal{S}$, there exists an $\mathcal{N}$-maximal element $\bar{\xi} \in \mathcal{S}$ satisfying $\xi_{0} \preceq \bar{\xi}$.

See Brezis-Browder [3] for the proof of Theorem 2.1] in case $\mathcal{N}$ is bounded from above and Cârjă-Ursescu [7] for the extension to the general case.

2.2. Excursion to functional analysis. In what follows, $X$ denotes a real Banach space $X$ with the norm $\|\cdot\|$. In this section we gather some results of functional analysis which will prove useful in the sequel.

Proposition 2.1. If $\lim _{n} x_{n}=x$ weakly in $X$, then there exists a sequence $\left(y_{n}\right)_{n}$, with $y_{n} \in \operatorname{conv}\left\{x_{k} ; k \geq n\right\}$ and such that $\lim _{n} y_{n}=x$.

See Hille-Phillips [16, Corollary to Theorem 2.9.3, p. 36. 
Definition 2.2. A function $x: \Omega \rightarrow X$ is called:

(i) countably-valued if there exist $\left\{\Omega_{n} ; n \in \mathbb{N}\right\} \subseteq \Sigma$ and $\left\{x_{n} ; n \in \mathbb{N}\right\} \subseteq X$, with $\Omega_{k} \cap \Omega_{p}=\emptyset$ for each $k \neq p, \Omega=\bigcup_{n \geq 0} \Omega_{n}$, and such that $x(\theta)=x_{n}$ for all $\theta \in \Omega_{n}$;

(ii) measurable if there exists a sequence of countably-valued functions convergent to $x \mu$-a.e. on $\Omega$.

Theorem 2.2. A function $x: \Omega \rightarrow X$ is measurable if and only if there exists a sequence of countably-valued functions from $\Omega$ to $X$ which is uniformly $\mu$-a.e. convergent on $\Omega$ to $x$.

See Vrabie 24], Theorem 1.1.3, p. 3 and Remark 1.1.2, p. 4.

Theorem 2.3. The closed convex hull of a (weakly) compact subset in a Banach space is (weakly) compact.

See Dunford-Schwartz [13, Theorem 6, p. 416 and Theorem 4, p. 434.

Theorem 2.4. A subset in a Banach space is weakly compact if and only if it is weakly sequentially compact.

See Edwards [14, Theorem 8.12.1, p. 549 and Theorem 8.12.7, p. 551.

Theorem 2.5. Let $X$ be reflexive. A subset in $X$ is weakly relatively sequentially compact if and only if it is norm-bounded.

See Hille-Phillips [16, Theorem 2.10.3, p. 38.

Theorem 2.6. Let $(\Omega, \Sigma, \mu)$ be a finite measure space and let $X$ be a Banach space. Let $\mathcal{F} \subseteq L^{1}(\Omega, \mu ; X)$ be bounded and uniformly integrable. If for each $\varepsilon>0$ there exist a weakly compact subset $C_{\varepsilon} \subseteq X$ and a measurable subset $E_{\varepsilon} \in \Sigma$ with $\mu\left(\Omega \backslash E_{\varepsilon}\right) \leq \varepsilon$ and $f\left(E_{\varepsilon}\right) \subseteq C_{\varepsilon}$ for all $f \in \mathcal{F}$, then $\mathcal{F}$ is weakly relatively compact in $L^{1}(\Omega, \mu ; X)$.

See Diestel [1], or Diestel-Uhl [12, p. 117.

Corollary 2.1. If $C \subseteq X$ is weakly compact, then

$$
\left\{f \in L^{1}(\tau, T ; X) ; f(t) \in C \text { a.e. for } t \in[\tau, T]\right\}
$$

is weakly relatively compact in $L^{1}(\tau, T ; X)$.

The next result is an infinite-dimensional version of the Arzełà-Ascoli Theorem.

Theorem 2.7. Let $X$ be a Banach space. A subset $\mathcal{F}$ in $C([\tau, T] ; X)$ is relatively compact if and only if:

(i) $\mathcal{F}$ is equicontinuous on $[\tau, T]$;

(ii) there exists a dense subset $D$ in $[\tau, T]$ such that, for each $t \in D, \mathcal{F}(t)=$ $\{f(t) ; f \in \mathcal{F}\}$ is relatively compact in $X$.

See Vrabie [24, Theorem A.2.1, p. 296.

2.3. Multi-functions. Let $K$ and $X$ be topological spaces and let $F: K \leadsto X$ be a given multi-function, i.e., a function $F: K \rightarrow 2^{X}$.

Definition 2.3. The multi-function $F: K \leadsto X$ is upper semicontinuous (u.s.c.) at $\xi \in K$ if for every open neighborhood $V$ of $F(\xi)$ there exists an open neighborhood $U$ of $\xi$ such that $F(\eta) \subseteq V$ for each $\eta \in U \cap K$. We say that $F$ is upper semicontinuous (u.s.c.) on $K$ if it is u.s.c. at each $\xi \in K$. 
Lemma 2.1. If $F: K \leadsto X$ is a nonempty and (weakly) compact valued, (stronglyweakly) u.s.c. multi-function, then, for each compact subset $C$ of $K, \bigcup_{\xi \in C} F(\xi)$ is (weakly) compact. In particular, in both cases, for each compact subset $C$ of $K$, there exists $M>0$ such that $\|\eta\| \leq M$ for each $\xi \in C$ and each $\eta \in F(\xi)$.

See Aubin-Cellina [1, Proposition 3, p. 42.

Lemma 2.2. Let $X$ be a Banach space and $K$ a nonempty subset in $X$. Let $F: K \leadsto X$ be a nonempty, closed and convex valued, strongly-weakly u.s.c. multifunction 11 and let $u_{m}:[0, T] \rightarrow K$ and $f_{m} \in L^{1}(0, T ; X)$ be such that $f_{m}(t) \in$ $F\left(u_{m}(t)\right)$ for each $m \in \mathbb{N}$ and a.e. for $t \in[0, T]$.

If $\lim _{m} u_{m}(t)=u(t)$ a.e. for $t \in[0, T]$ and $\lim _{m} f_{m}=f$ weakly in $L^{1}(0, T ; X)$, then $f(t) \in F(u(t))$ a.e. for $t \in[0, T]$.

See Vrabie [23], Theorem 3.1.2, p. 88.

2.4. Measure of noncompactness. Let $X$ be a Banach space and let $\mathcal{B}(X)$ be the family of all bounded subsets of $X$.

Definition 2.4. The function $\beta: \mathcal{B}(X) \rightarrow \mathbb{R}_{+}$, defined by

$$
\beta(B)=\inf \left\{\varepsilon>0 ; \exists x_{1}, x_{2}, \ldots, x_{n(\varepsilon)} \in X, B \subseteq \bigcup_{i=1}^{n(\varepsilon)} D\left(x_{i}, \varepsilon\right)\right\}
$$

is called the Hausdorff measure of noncompactness on $X$.

Proposition 2.2. Let $Y \subseteq X$ be a subspace in $X$ and let us define $\beta_{Y}: \mathcal{B}(X) \rightarrow \mathbb{R}_{+}$ by

$$
\beta_{Y}(B)=\inf \left\{\varepsilon>0 ; \exists x_{1}, x_{2}, \ldots, x_{n(\varepsilon)} \in Y, B \subseteq \bigcup_{i=1}^{n(\varepsilon)} D\left(x_{i}, \varepsilon\right)\right\} .
$$

Then

$$
\beta(B) \leq \beta_{Y}(B) \leq 2 \beta(B) .
$$

See Mönch [17, Proposition 1.3.

Definition 2.5. The Hausdorff-Pompeiu distance between the sets $B, C \in \mathcal{B}(X)$ is defined by $\operatorname{dist}_{H P}(B, C)=\max \{e(B ; C), e(C ; B)\}$, where, for each $B, C \in \mathcal{B}(X)$, $e(B ; C)$ is the excess of $B$ over $C$, defined by $e(B ; C)=\sup _{x \in B} \operatorname{dist}(x ; C)$.

Proposition 2.3. We have:

(i) $\beta(B)=\beta(\bar{B})$;

(ii) $\beta(B)=0$ if and only if $B$ is relatively compact;

(iii) $\beta(\lambda B) \leq|\lambda| \beta(B)$ for each $\lambda \in \mathbb{R}$ and $B \in \mathcal{B}(X)$;

(iv) $\beta(B+C) \leq \beta(B)+\beta(C)$ for each $B, C \in \mathcal{B}(X)$;

(v) if $B \subseteq C$, then $\beta(B) \leq \beta(C)$;

(vi) $\beta(B \cup C)=\max \{\beta(B), \beta(C)\}$;

(vii) $\beta(\operatorname{conv}(B))=\beta(B)$;

(viii) $\beta$ is nonexpansive with respect to the Hausdorff-Pompeiu distance. More precisely, $|\beta(B)-\beta(C)| \leq \operatorname{dist}_{H P}(B ; C)$ for each $B, C \in \mathcal{B}(X)$.

See Deimling [10, Proposition 7.2, p. 41.

\footnotetext{
${ }^{1}$ Of course if $F$ is strongly-strongly u.s.c. it is strongly-weakly u.s.c. too, and thus the conclusion of Lemma 2.2 holds true also in this case.
} 
Lemma 2.3. Let $X$ be a separable Banach space and $\left\{F_{m} ; m \in \mathbb{N}\right\}$ a subset in $L^{1}(\tau, T ; X)$ for which there exists $\ell \in L^{1}\left(\tau, T ; \mathbb{R}_{+}\right)$such that

$$
\left\|F_{m}(s)\right\| \leq \ell(s)
$$

for each $m \in \mathbb{N}$ and a.e. for $s \in[\tau, T]$. Then the mapping

$$
s \mapsto \beta\left(\left\{F_{m}(s) ; m \in \mathbb{N}\right\}\right)
$$

is integrable on $[\tau, T]$, and, for each $t \in[\tau, T]$, we have

$$
\beta\left(\left\{\int_{\tau}^{t} F_{m}(s) d s ; m \in \mathbb{N}\right\}\right) \leq \int_{\tau}^{t} \beta\left(\left\{F_{m}(s) ; m \in \mathbb{N}\right\}\right) d s
$$

See Mönch [17.

Remark 2.1. If $X$ is not separable, there is a simple trick which may very often be useful. Namely, let $\left\{F_{m} ; m \in \mathbb{N}\right\}$ be a subset in $L^{1}(\tau, T ; X)$ for which there exists $\ell \in L^{1}\left(\tau, T ; \mathbb{R}_{+}\right)$such that

$$
\left\|F_{m}(s)\right\| \leq \ell(s)
$$

for each $m \in \mathbb{N}$ and a.e. for $s \in[\tau, T]$. In view of Theorem 2.2, there exists a separable and closed subspace $Y$ of $X$ such that $F_{m} \in L^{1}(\tau, T ; Y)$ for $m=1,2, \ldots$. Let us observe that the restriction of the mapping $\beta_{Y}$ (see Proposition 2.2) to $\mathcal{B}(Y)$ coincides with the Hausdorff measure of noncompactness on $Y$. Then, from Lemma 2.3, it follows that the function $s \mapsto \beta_{Y}\left(\left\{F_{m}(s) ; m \in \mathbb{N}\right\}\right)$ is integrable on $[\tau, T]$ and, for each $t \in[\tau, T]$, we have

$$
\beta_{Y}\left(\left\{\int_{\tau}^{t} F_{m}(s) d s ; m \in \mathbb{N}\right\}\right) \leq \int_{\tau}^{t} \beta_{Y}\left(\left\{F_{m}(s) ; m \in \mathbb{N}\right\}\right) d s .
$$

We conclude this section with

Lemma 2.4. Let $\left(u_{n}\right)_{n}$ be a bounded sequence in $X$ such that

$$
\lim _{k} \beta\left(\left\{u_{n} ; n \geq k\right\}\right)=0 .
$$

Then $\left\{u_{n} ; n \in \mathbb{N}\right\}$ is relatively compact.

Proof. The conclusion follows from the remark that, for each $k, p \in \mathbb{N}$, we have

$$
\beta\left(\left\{u_{n} ; n \geq k\right\}\right)=\beta\left(\left\{u_{n} ; n \geq p\right\}\right) .
$$

\subsection{Integral inequalities.}

Definition 2.6. A function $\omega: \mathbb{R}_{+} \rightarrow \mathbb{R}_{+}$which is continuous, nondecreasing and for which the only $C^{1}$-solution of the Cauchy problem

$$
\left\{\begin{array}{l}
x^{\prime}(t)=\omega(x(t)) \\
x(0)=0
\end{array}\right.
$$

is $x \equiv 0$ is called a uniqueness function.

Remark 2.2. If $\omega: \mathbb{R}_{+} \rightarrow \mathbb{R}_{+}$is a uniqueness function, then, for each $m>0, m \omega$ is a uniqueness function too. Similarly, if $m>0, x \mapsto \omega(m x)$ is a uniqueness function too. 
Lemma 2.5. Let $\omega: \mathbb{R}_{+} \rightarrow \mathbb{R}_{+}$be a uniqueness function and let $\left(\gamma_{k}\right)_{k}$ be decreasing to 0 . Let $\left(x_{k}\right)_{k}$ be a bounded sequence of measurable functions, from $\left[0, T_{1}\right]$ to $\mathbb{R}_{+}$, such that

$$
x_{k}(t) \leq \gamma_{k}+\int_{0}^{t} \omega\left(x_{k}(s)\right) d s
$$

for $k=1,2, \ldots$ and for each $t \in\left[0, T_{1}\right]$. Then there exists $T \in\left(0, T_{1}\right]$ such that $\lim _{k} x_{k}(t)=0$ uniformly for $t \in[0, T]$.

The proof is standard and so we omit it.

\section{TANGENCY CONCEPTS}

3.1. Tangent sets. Let $C$ and $D$ be nonempty subsets in $X$ and let $z \in X$. We denote by dist $(C ; D)$ the usual distance between $C$ and $D$, i.e.,

$$
\operatorname{dist}(C ; D)=\inf _{x \in C, y \in D}\|x-y\| .
$$

We will also use the following notation:

$$
\operatorname{dist}(z ; C)=\inf _{y \in C}\|z-y\|
$$

and

$$
z+C=\{y \in X ; \text { there exists } w \in C \text { such that } y=z+w\} .
$$

Definition 3.1. Let $K \subseteq X$ and $\xi \in K$. The set $E \subseteq X$ is tangent to the set $K$ at the point $\xi$ if, for each $\rho>0$, we have

$$
\liminf _{h \downarrow 0} \frac{1}{h} \operatorname{dist}(\xi+h E ; K \cap D(\xi, \rho))=0 .
$$

We denote by $\mathcal{T S}_{K}(\xi)$ the class of all sets which are tangent to $K$ at the point $\xi$.

One may easily verify that if $K$ is open, then, for each $\xi \in K, \mathcal{T S}_{K}(\xi)$ consists of all nonempty subsets in $X$.

The next two propositions follow easily from Definition 3.1

Proposition 3.1. Let $K \subseteq X, \xi \in K$ and $E \subseteq X$. Then, the following conditions are equivalent:

(i) $E \in \mathcal{T S}_{K}(\xi)$;

(ii) there exist two sequences, $\left(h_{n}\right)_{n}$ in $\mathbb{R}_{+}$with $h_{n} \downarrow 0$ and $\left(\eta_{n}\right)_{n}$ in $E$ such that $\lim _{n} h_{n} \eta_{n}=0$, and $\liminf _{n} \frac{1}{h_{n}} \operatorname{dist}\left(\xi+h_{n} \eta_{n} ; K\right)=0$;

(iii) for each $\varepsilon>0, \rho>0$ and $\delta>0$ there exist $h \in(0, \delta), p \in D(0, \varepsilon)$ and $\eta \in E$ such that $\xi+h \eta+h p \in K \cap D(\xi, \rho)$;

(iv) there exist three sequences, $\left(h_{n}\right)_{n}$ in $\mathbb{R}_{+}$with $h_{n} \downarrow 0,\left(\eta_{n}\right)_{n}$ in $E$ with $\lim _{n} h_{n} \eta_{n}=0$ and $\left(p_{n}\right)_{n}$ in $X$, with $\lim _{n} p_{n}=0$, such that $\xi+h_{n} \eta_{n}+h_{n} p_{n} \in$ $K$ for $n=1,2, \ldots$.

Let us denote by $\mathcal{B}(X)$ the class of all bounded subsets in $X$.

Proposition 3.2. Let $K \subseteq X, \xi \in K$ and $E \in \mathcal{B}(X)$. Then, the following conditions are equivalent:

(i) $E \in \mathcal{T S}_{K}(\xi)$;

(ii) $\liminf \operatorname{in}_{h \downarrow} \frac{1}{h} \operatorname{dist}(\xi+h E ; K)=0$;

(iii) there exist two sequences, $\left(h_{n}\right)_{n}$ in $\mathbb{R}_{+}$with $h_{n} \downarrow 0$ and $\left(\eta_{n}\right)_{n}$ in $E$, such that $\liminf _{n} \frac{1}{h_{n}} \operatorname{dist}\left(\xi+h_{n} \eta_{n} ; K\right)=0$; 
(iv) for each $\varepsilon>0$ there exist $\eta \in E, \delta \in(0, \varepsilon)$ and $p \in X$ with $\|p\| \leq \varepsilon$, such that $\xi+\delta \eta+\delta p \in K$

(v) there exist three sequences, $\left(h_{n}\right)_{n}$ in $\mathbb{R}_{+}$with $h_{n} \downarrow 0,\left(\eta_{n}\right)_{n}$ in $E$ and $\left(p_{n}\right)_{n}$ in $X$ with $\lim _{n} p_{n}=0$, such that $\xi+h_{n} \eta_{n}+h_{n} p_{n} \in K$ for $n=1,2, \ldots$

Remark 3.1. One may ask why we did not choose to define the concept of tangent set by merely imposing (ii) in Proposition 3.2 instead of (3.1). See Definition 3.1. To answer this question, let us observe that, whenever $E$ is unbounded, (ii) in Proposition 3.2 does not ensure a local character to the tangency concept, i.e. $\mathcal{T S}_{K}(\xi)=\mathcal{T S}_{K \cap D(\xi, \rho)}(\xi)$ for each $\rho>0$. See the example below.

Example 3.1. Let us consider $X=\mathbb{R}^{2}, K=K_{1} \cup K_{2}$, where

$$
\left\{\begin{array}{l}
K_{1}=\left\{(x, y) \in \mathbb{R}^{2} ; y \leq 0, x^{2}+y^{2} \geq 1\right\} \\
K_{2}=\left\{(x, y) \in \mathbb{R}^{2} ; x=0, y \in[-1,0]\right\}
\end{array}\right.
$$

and $\xi=(0,0) \in K$. Let

$$
E=\left\{(x, y) \in \mathbb{R}^{2} ; y \geq 0, x^{2}+y^{2} \geq 1\right\} .
$$

Since dist $(\xi+h E ; K)=\operatorname{dist}(h E ; K)=0, E, K$ and $\xi$ satisfy (ii) in Proposition 3.2, However, for every $\rho \in(0,1)$,

$$
\liminf _{h \downarrow 0} \frac{1}{h} \operatorname{dist}(\xi+h E ; K \cap D(\xi, \rho))=1 .
$$

Proposition 3.3. Let $K \subseteq X, \xi \in K$ and $E \in \mathcal{B}(X)$. Then, we have:

(i) if $0 \in E$, then $E \in \mathcal{T S}_{K}(\xi)$;

(ii) $\{0\} \in \mathcal{T S}_{K}(\xi)$;

(iii) if $E \in \mathcal{T S}_{K}(\xi)$ and $E \subseteq D$, then $D \in \mathcal{T} \mathcal{S}_{K}(\xi)$;

(iv) $E \in \mathcal{T S}_{K}(\xi)$ if and only if $\bar{E} \in \mathcal{T S}_{K}(\xi)$;

(v) if $E \in \mathcal{T S}_{K}(\xi)$, then for each $\lambda>0$, we have $\lambda E \in \mathcal{T S}_{K}(\xi)$;

(vi) for each $\xi \in K$, the set $\mathcal{T S}_{K}(\xi)$ is closed from the left with respect to the excess e; i.e., if $E \in \mathcal{B}(X)$ and $\left(E_{n}\right)_{n}$ is a sequence in $\mathcal{T S}_{K}(\xi)$ such that $\lim _{n} e\left(E_{n} ; E\right)=0$, then $E \in \mathcal{T S}_{K}(\xi)$. In particular, for each $\xi \in K$, the set $\mathcal{T S}_{K}(\xi)$ is closed with respect to the Hausdorff-Pompeiu distance.

Proof. Since (i) $\sim(v)$ are simple consequences of Definition 3.1 we confine ourselves only to the proof of (vi). To check (vi), let $E \in \mathcal{B}(X)$ and let $\left(E_{n}\right)_{n}$ be such that $E_{n} \in \mathcal{T S}_{K}(\xi)$ for $n=1,2, \ldots$ and

$$
\lim _{n} e\left(E_{n} ; E\right)=0 .
$$

Let $\varepsilon>0$ and fix $n=1,2, \ldots$ such that

$$
e\left(E_{n} ; E\right) \leq \varepsilon .
$$

Since $E_{n} \in \mathcal{T S}_{K}(\xi)$, in view of the equivalence of (i) and (ii) in Proposition 3.2, there exist $\widetilde{\eta}_{n} \in E_{n}$ and $h_{n} \in(0, \varepsilon)$ such that

$$
\operatorname{dist}\left(\xi+h_{n} \widetilde{\eta}_{n} ; K\right) \leq h_{n} \varepsilon \text {. }
$$

Since $e\left(E_{n} ; E\right) \leq \varepsilon$, there exists $\eta_{n} \in E$ such that $\left\|\eta_{n}-\widetilde{\eta}_{n}\right\| \leq 2 \varepsilon$. We then have

$$
\operatorname{dist}\left(\xi+h_{n} \eta_{n} ; K\right) \leq \operatorname{dist}\left(\xi+h_{n} \widetilde{\eta}_{n} ; K\right)+h_{n}\left\|\widetilde{\eta}_{n}-\eta_{n}\right\| \leq 3 h_{n} \varepsilon .
$$

But this inequality combined with (iii) in Proposition 3.2 shows that $E \in \mathcal{T S}_{K}(\xi)$ and this completes the proof. 
3.2. Bouligand-Severi tangent vectors. We recall next the definition of the tangent vector to a set at a given point as introduced independently and at the very same time by Bouligand [4] and Severi [21].

Definition 3.2. Let $K \subseteq X$ and $\xi \in K$. The vector $\eta \in X$ is tangent in the sense of Bouligand-Severi to the set $K$ at the point $\xi$ if

$$
\liminf _{h \downarrow 0} \frac{1}{h} \operatorname{dist}(\xi+h \eta ; K)=0 .
$$

We denote by $\mathcal{T}_{K}(\xi)$ the set of all vectors which are tangent in the sense of Bouligand-Severi to the set $K$ at the point $\xi$.

Remark 3.2. It is easy to prove that for each $\xi \in K$, the set $\mathcal{T}_{K}(\xi)$ is a closed cone. The cone $\mathcal{T}_{K}(\xi)$ is called the contingent cone to $K$ at $\xi$.

The following proposition follows easily from Definition 3.2 .

Proposition 3.4. A vector $\eta \in X$ belongs to the cone $\mathcal{T}_{K}(\xi)$ if and only if for every $\varepsilon>0$ there exist $h \in(0, \varepsilon)$ and $p \in D(0, \varepsilon)$ with the property

$$
\xi+h(\eta+p) \in K \text {. }
$$

Corollary 3.1. A vector $\eta \in X$ belongs to the cone $\mathcal{T}_{K}(\xi)$ if and only if there exist two sequences $\left(h_{m}\right)_{m}$ in $\mathbb{R}_{+}$and $\left(p_{m}\right)_{m}$ in $X$ with $h_{m} \downarrow 0, \lim _{m} p_{m}=0$ and such that $\xi+h_{m}\left(\eta+p_{m}\right) \in K$ for each $m \in \mathbb{N}$.

Remark 3.3. We notice that, if $\xi$ is an interior point of the set $K$, then $\mathcal{T}_{K}(\xi)=X$. Indeed, in this case there exists $\rho>0$ with $D(\xi, \rho) \subset K$ and, therefore, for $t>0$ sufficiently small, $\xi+t \eta \in D(\xi, \rho) \subseteq K$. Obviously, for such numbers $t>0$, we have dist $(\xi+t \eta ; K)=0$, from which the condition in Definition 3.2 follows.

Remark 3.4. By the natural injection $\eta \mapsto\{\eta\}, \mathcal{T}_{K}(\xi)$ is identified with a subclass of $\mathcal{T S}_{K}(\xi)$. Therefore, in the sequel, by $\mathcal{T}_{K}(\xi) \subseteq \mathcal{T S}_{K}(\xi)$ we mean the natural inclusion induced by the injection above.

Remark 3.5. If $K$ is a closed cone, then $\mathcal{T}_{K}(0)=K$.

The next proposition shows that in case the set $E$ is compact, the property that $E$ is tangent to the set $K$ at the point $\xi \in K$ can be characterized in terms of tangent vectors.

Proposition 3.5. Let $K \subseteq X$ and $\xi \in K$ be arbitrary and let $E$ be compact. Then $E \in \mathcal{T S}_{K}(\xi)$ if and only if $E \cap \mathcal{T}_{K}(\xi) \neq \emptyset$.

Proof. Let $E \in \mathcal{T S}_{K}(\xi)$ be compact. Then we may assume with no loss of generality that $\left(\eta_{n}\right)_{n}$ in $(\mathrm{v})$, Proposition 3.2 is convergent to some $\eta \in E$. We then have $\xi+h_{n} \eta_{n}+h_{n} p_{n} \in K$ if and only if $\xi+h_{n} \eta+h_{n} q_{n} \in K$, where $q_{n}=p_{n}+\eta_{n}-\eta \rightarrow 0$. In view of Proposition $3.2,\{\eta\} \in \mathcal{T S}_{K}(\xi)$, i.e. $\eta \in \mathcal{T}_{K}(\xi)$.

Remark 3.6. In order to establish that the new concept of tangent set is nontrivial, we have first to ask whether or not there are tangent sets $E$ which do not contain tangent vectors in the sense of Bouligand-Severi. The answer to this question is in the affirmative, as shown by the Examples 3.2 and 3.3 below. Namely, there exist $X, K \subseteq X, \xi \in K$ and a weakly compact set $E \subseteq X$, with $E \in \mathcal{T S}_{K}(\xi)$ but $E \cap \mathcal{T}_{K}(\xi)=\emptyset$. 
Example 3.2. Let $X=\ell_{2}$ be the space of all real sequences $\left(x_{k}\right)_{k}$, with $\sum_{k=1}^{\infty} x_{k}^{2}<$ $\infty$, endowed with its usual norm $\|\cdot\|$, defined by

$$
\left\|\left(x_{k}\right)_{k}\right\|=\left(\sum_{k=1}^{\infty} x_{k}^{2}\right)^{1 / 2}
$$

for each $\left(x_{k}\right)_{k} \in \ell_{2}$. Let $\left\{e_{1}, e_{2}, \ldots\right\}$ be the standard orthonormal basis in $\ell_{2}, \xi \in \ell_{2}$ with $\|\xi\|=2$, let $\left(h_{k}\right)_{k}$ be a sequence in $(0,1], h_{k} \downarrow 0, f_{n}=\xi+e_{n}$, for $n=1,2, \ldots$, let $E=\xi+D(0,1)$ and $K=\left\{h_{n} f_{n} ; n=1,2, \ldots\right\} \cup\{0\}$. Clearly $K$ is compact, $f_{n} \in E$, for $n=1,2, \ldots$, and

$$
\operatorname{dist}\left(h_{n} E ; K\right)=0,
$$

for $n=1,2, \ldots$ Thus $E \in \mathcal{T S}_{K}(0)$. However, $\mathcal{T}_{K}(0)=\{0\}$. Indeed, the inclusion $\{0\} \subseteq \mathcal{T}_{K}(0)$ is obvious. Now, if we assume by contradiction that there exists $\eta \in \ell_{2}, \eta \neq 0$, with $\eta \in \mathcal{T}_{K}(0)$, then there would exist $\left(t_{n}\right)_{n}$ in $(0,1)$ with $t_{n} \downarrow 0$ and a sequence of natural numbers $\left(k_{n}\right)_{n}$, such that

$$
\lim _{n} \frac{1}{t_{n}}\left\|t_{n} \eta-h_{k_{n}} f_{k_{n}}\right\|=0
$$

or equivalently

$$
\lim _{n}\left\|\eta-\frac{h_{k_{n}}}{t_{n}} f_{k_{n}}\right\|=0
$$

Let us observe that $\left(k_{n}\right)_{n}$ cannot have constant subsequences. Indeed, if we assume that $\left(k_{n}\right)_{n}$ has a constant subsequence, denoted for simplicity again by $\left(k_{n}\right)_{n}=$ $(\widetilde{k})_{n}$, then, since $\left\|f_{\widetilde{k}}\right\|=\left\|\xi+e_{\widetilde{k}}\right\| \geq\|\xi\|-\left\|e_{\widetilde{k}}\right\| \geq 1$, we deduce

$$
\lim _{n}\left\|\eta-\frac{h_{\widetilde{k}}}{t_{n}} f_{\widetilde{k}}\right\| \geq \lim _{n}\left(\frac{h_{\widetilde{k}}}{t_{n}}\left\|f_{\widetilde{k}}\right\|-\|\eta\|\right)=\infty,
$$

which contradicts (3.3). Therefore we have $k_{n} \rightarrow \infty$ as $n \rightarrow \infty$.

A similar argument shows that $\left(\frac{h_{k_{n}}}{t_{n}}\right)_{n}$ is necessarily bounded. Hence, we may assume, without loss of generality, that it is convergent. In addition,

$$
\lim _{n} \frac{h_{k_{n}}}{t_{n}}=m>0,
$$

because otherwise, in view of (3.3), we would get a contradiction, i.e., $\|\eta\|=0$. Therefore, again by (3.3), we get $\lim _{n}\left\|\eta-m f_{k_{n}}\right\|=0$, which shows that $f_{k_{n}} \rightarrow \frac{1}{m} \eta$. But this is impossible because $\left(f_{n}\right)_{n}$ cannot have strongly convergent subsequences. To justify the last assertion, it suffices to observe that $\left\|f_{i}-f_{j}\right\|=\sqrt{2}$, for $i, j=$ $1,2, \ldots, i \neq j$. The contradiction $f_{k_{n}} \rightarrow \frac{1}{m} \eta$ can be eliminated only if $\eta \notin \mathcal{T}_{K}(0)$. Thus $\mathcal{T}_{K}(0)=\{0\}$ and since each $f \in E$ satisfies $\|f\| \geq 1, E \cap \mathcal{T}_{K}(0)=\emptyset$, as claimed.

Example 3.2 can be refined to allow $K$ to be a noncompact, closed cone.

Example 3.3. Let $X=\ell_{2}$ and $\left\{e_{1}, e_{2}, \ldots\right\}$ be as in Example 3.2, let $y=\left(y_{k}\right)_{k} \in \ell_{2}$ be such that $y_{k}>0$ for $k=1,2, \ldots$ and let

$$
E=y+\overline{\operatorname{conv}}\left\{e_{n} ; n=1,2, \ldots\right\} .
$$


Let $f_{n}=y+e_{n}+\frac{1}{n} e_{n}, n=1,2, \ldots$, and let us define

$$
K=\left\{\lambda f_{n} ; \lambda \geq 0, n=1,2, \ldots\right\} .
$$

We have $E \in \mathcal{T S}_{K}(0)$ and $E \cap \mathcal{T}_{K}(0)=\emptyset$.

We also may allow $X$ to be nonreflexive.

Example 3.4. Let $X=C([0,1])$, which, endowed with the usual sup-norm, defined by $\|f\|=\sup _{t \in[0,1]}|f(t)|$ for $f \in C([0,1])$, is a nonreflexive Banach space. Let

$$
\begin{gathered}
K=\{f \in C([0,1]) ; \text { there exists } t \in[0,1] \text { with } f(t) \leq 0\}, \\
E=\{f \in C([0,1]) ; t \leq f(t) \leq 1 \text { for all } t \in[0,1] \text { and } f(0)=f(1)=1\} .
\end{gathered}
$$

Let $\xi=0 \in K$. Then, $E \in \mathcal{T S}_{K}(\xi)$ but, nevertheless, $E \cap \mathcal{T}_{K}(\xi)=\emptyset$.

The set $E$ in the last example is not weakly compact. It should be interesting to answer the question raised in the open problem below.

Open problem. Prove or disprove that in each infinite-dimensional Banach space there exist a set $K$, a point $\xi \in K$, and a weakly compact subset $E$ such that $E \in \mathcal{T S}_{K}(\xi)$ but $E \cap \mathcal{T}_{k}(\xi)=\emptyset$.

\section{NECESSARY CONDITIONS FOR EXACT VIABILITY}

4.1. Exact solutions. Let $X$ be a Banach space, $K$ a nonempty subset in $X$, $F: K \leadsto X$ a given multi-function and let us consider the Cauchy problem

$$
\left\{\begin{array}{l}
u^{\prime}(t) \in F(u(t)) \\
u(0)=\xi
\end{array}\right.
$$

Definition 4.1. An exact solution of (4.1) on $[0, T]$ is an absolutely continuous function $u:[0, T] \rightarrow K$ which is a.e. differentiable on $[0, T]$ with $u^{\prime} \in L^{1}(0, T ; X)$ and satisfies

$$
\left\{\begin{array}{l}
u^{\prime}(t) \in F(u(t)) \quad \text { at each point } t \in[0, T] \text { at which } u \text { is differentiable } \\
u(0)=\xi
\end{array}\right.
$$

An exact solution of (4.1) on the semi-open interval $[0, T)$ is defined similarly, by noticing that, in this case, we have merely to impose that $u^{\prime} \in L_{\mathrm{loc}}^{1}([0, T) ; X)$.

Definition 4.2. An almost exact solution of (4.1) on $[0, T]$ is an absolutely continuous function $u:[0, T] \rightarrow K$ which is a.e. differentiable on $[0, T]$ with $u^{\prime} \in$ $L^{1}(0, T ; X)$ and satisfies

$$
\left\{\begin{array}{l}
u^{\prime}(t) \in F(u(t)) \quad \text { a.e. for } t \in[0, T] \\
u(0)=\xi
\end{array}\right.
$$

An almost exact solution of (4.1) on the semi-open interval [ $0, T)$ is defined similarly, by noticing that, in this case, we have merely to impose that $u^{\prime} \in L_{\mathrm{loc}}^{1}([0, T) ; X)$.

Remark 4.1. Clearly each exact solution is almost exact. It should be emphasized that, although not obvious, under some very natural continuity assumptions on $F$, the converse statement is also true as we shall later prove. See Corollary 4.1 below. 
Remark 4.2. If $u$ is an almost exact solution of (4.1) on $[0, T]$, we have

$$
u(t)=u(s)+\int_{s}^{t} u^{\prime}(\theta) d \theta
$$

for each $0 \leq s \leq t \leq T$. In view of Remark 4.1 the equality above also holds true if $u$ is an exact solution.

Definition 4.3. The set $K$ is exact viable (almost exact viable) with respect to $F$ if for each $\xi \in K$ there exists $T>0$ such that (4.1) has at least one exact solution (almost exact solution) $u:[0, T] \rightarrow K$.

Let $u:[0, T] \rightarrow X$ and let $t \in[0, T)$. We denote by $\mathcal{D}_{+} u(t)$ the set of all limit points of the mapping $h \mapsto h^{-1}(u(t+h)-u(t))$ for $h \downarrow 0$. Clearly, if $u$ is right differentiable at $t$ and $u_{+}^{\prime}(t)$ is the right derivative of $u$ at $t$, we have $\mathcal{D}_{+} u(t)=\left\{u_{+}^{\prime}(t)\right\}$. In particular, if $u$ is differentiable at $t$, then $\mathcal{D}_{+} u(t)=\left\{u^{\prime}(t)\right\}$.

Theorem 4.1. Let $X$ be a Banach space, let $F: K \leadsto X$ be a strongly-weakly u.s.c. multi-function with nonempty, closed and convex values and let $u:[0, T] \rightarrow K$ be an almost exact solution of (4.1). Then

$$
\mathcal{D}_{+} u(t) \subseteq F(u(t)) \cap \mathcal{T}_{K}(u(t))
$$

for each $t \in[0, T)$.

Proof. Let $t \in[0, T)$ be such that the mapping $h \mapsto h^{-1}(u(t+h)-u(t))$ has no limit point for $h \downarrow 0$. Then $\mathcal{D}_{+} u(t)=\emptyset$, and we have nothing to prove. So, let $t \in[0, T)$ be such that $\mathcal{D}_{+} u(t)$ is nonempty and let $\eta \in \mathcal{D}_{+} u(t)$ be arbitrary. Then there exists $h_{k} \downarrow 0$ such that

$$
\lim _{k}\left(h_{k}\right)^{-1}\left(u\left(t+h_{k}\right)-u(t)\right)=\eta .
$$

As $F$ is strongly-weakly u.s.c. at $u(t)$ and $s \mapsto u(s)$ is continuous, we conclude that for each open half space $V$ with $F(u(t)) \subseteq V$, there exists a positive integer $k(V)$ such that, for $k=k(V), k(V)+1, \ldots$, we have

$$
\frac{1}{h_{k}}\left(u\left(t+h_{k}\right)-u(t)\right)=\frac{1}{h_{k}} \int_{t}^{t+h_{k}} u^{\prime}(s) d s \in \bar{V} .
$$

Thus $\eta \in \bar{V}$. But $F(u(t))$ is closed and convex. Hence, it is the intersection of all closed half spaces which contain it. So $\eta \in F(u(t))$. To complete the proof, we have merely to observe that

$$
\lim _{k} \frac{1}{h_{k}} \operatorname{dist}\left(u(t)+h_{k} \eta ; K\right) \leq \lim _{k} \frac{1}{h_{k}}\left\|u(t)+h_{k} \eta-u\left(t+h_{k}\right)\right\|=0,
$$

which shows that $\eta \in \mathcal{T}_{K}(u(t))$. Since $\eta \in \mathcal{D}_{+} u(t)$ was arbitrary, we deduce that $\mathcal{D}_{+} u(t) \subseteq F(u(t)) \cap \mathcal{T}_{K}(u(t))$, and this completes the proof.

Corollary 4.1. Let $X$ be a Banach space, let $F: K \leadsto X$ be a strongly-weakly u.s.c. multi-function with nonempty, closed and convex values. Then each almost exact solution of (4.1) is an exact solution too.

Corollary 4.2. Let $X$ be a Banach space, let $F: K \leadsto X$ be a strongly-weakly u.s.c. multi-function with nonempty, closed and convex values and let $u:[0, T] \rightarrow K$ be an exact solution of (4.1). If $t \in[0, T)$ is such that

$$
F(u(t)) \cap \mathcal{T}_{K}(u(t))=\emptyset,
$$

then $h \mapsto h^{-1}(u(t+h)-u(t))$ has no limit point for $h \downarrow 0$. 
Proposition 4.1. Let $X$ be a Banach space, let $F: K \leadsto X$ be a strongly-weakly u.s.c. multi-function with nonempty, closed and convex values. If $K$ is exact viable with respect to $F: K \leadsto X$, then $C=\left\{\xi \in K ; F(\xi) \cap \mathcal{T}_{K}(\xi) \neq \emptyset\right\}$ is dense in $K$.

Proof. Let $\xi \in K$. Let $u:[0, T] \rightarrow K$ be an exact solution of (4.1). Then, in view of Theorem 4.1, we have $u^{\prime}(t) \in F(u(t)) \cap \mathcal{T}_{K}(u(t))$ at each point of differentiability of $u$. Thus, there exists at least one sequence $\left(t_{k}\right)_{k}$ of differentiability points of $u$ such that $\lim _{k} t_{k}=0$. Consequently, we have $\lim _{k} u\left(t_{k}\right)=\xi$ and this completes the proof.

4.2. Necessary conditions for exact viability. The next general result is due to Marius Popescu (personal communication).

Theorem 4.2. Let $X$ be a Banach space. If $K \subseteq X$ is almost exact viable with respect to the multi-function $F: K \leadsto X$, then for each point $\xi \in K$ with $F(\xi)$ convex and at which $F$ is u.s.c., we have

$$
F(\xi) \in \mathcal{T S}_{K}(\xi) .
$$

Proof. Let $\xi \in K$ be a point with $F(\xi)$ convex and at which $F$ is u.s.c. Since $K$ is almost exact viable with respect to $F$, there exists at least one almost exact solution $u:[0, T] \rightarrow K$ of (4.1). As $u$ is continuous at $t=0, F$ is u.s.c. at $u(0)=\xi$ and $u^{\prime}(s) \in F(u(s))$, a.e. for $s \in[0, T]$, it follows that for each $\varepsilon>0, \rho>0$ and $\delta>0$ there exists $h \in(0, \delta)$ such that

$$
u^{\prime}(s) \in F(\xi)+D(0, \varepsilon)
$$

a.e. for $s \in[0, h]$ and

$$
u(s) \in K \cap D(\xi, \rho)
$$

for each $s \in[0, h]$. So,

$$
\frac{1}{h}(u(h)-\xi)=\frac{1}{h} \int_{0}^{h} u^{\prime}(s) d s \in \overline{\operatorname{conv}}(F(\xi)+D(0, \varepsilon)) .
$$

Since both $F(\xi)$ and $D(0, \varepsilon)$ are convex, it follows that

$$
\overline{\operatorname{conv}}(F(\xi)+D(0, \varepsilon))=\overline{F(\xi)+D(0, \varepsilon)} .
$$

Therefore

$$
\frac{1}{h}(u(h)-\xi) \in \overline{F(\xi)+D(0, \varepsilon)}
$$

and, accordingly, there exist $\eta \in F(\xi), q \in D(0, \varepsilon)$ and $r \in D(0, \varepsilon)$ such that

$$
u(h)=\xi+h \eta+h p,
$$

where $p=q+r$. Consequently, for each $\varepsilon>0, \rho>0$ and $\delta>0$ there exist $h \in(0, \delta), \eta \in F(\xi)$ and $p \in D(0,2 \varepsilon)$ such that $\xi+h \eta+h p \in K \cap D(\xi, \rho)$. In view of the equivalence of (i) and (iii) in Proposition 3.1. it follows that $F(\xi) \in \mathcal{T S}_{K}(\xi)$ and this completes the proof.

In the case in which $F$ is compact-valued, we get a necessary condition involving the contingent cone.

Theorem 4.3. If the set $K$ is almost exact viable with respect to the multi-function $F: K \leadsto X$, then for each point $\xi \in K$ with $F(\xi)$ convex and compact and at which $F$ is u.s.c., we have $F(\xi) \cap \mathcal{T}_{K}(\xi) \neq \emptyset$.

Proof. Combine Theorem 4.2 and Proposition 3.5 . 


\section{SUfFICIENT CONDITIONS FOR EXACT VIABILITY}

Let $X$ and $Y$ be Banach spaces, $D$ a nonempty subset in $Y$ and $F: D \leadsto X$ a given multi-function.

Definition 5.1. A multi-function $F: D \leadsto X$ is locally compact if it is u.s.c. and for each $\eta \in D$ there exists $\rho>0$ such that $F\left(D_{Y}(\eta, \rho) \cap D\right)$ is relatively compact in $X$. Further, $F$ is called compact if it is u.s.c. and carries bounded subsets in $D$ into relatively compact subsets in $X$.

Definition 5.2. Let $Y$ and $X$ be two Banach spaces and let $D \subseteq Y$. A multifunction $F: D \leadsto X$ is called locally $\beta$-compact if it is u.s.c. and, for each $y \in D$, there exist $r>0$ and a uniqueness function $\omega: \mathbb{R}_{+} \rightarrow \mathbb{R}_{+}$such that for each subset $C$ in $D_{Y}(y, r) \cap D, F(C)$ is bounded, and we have

$$
\beta_{X}(F(C)) \leq \omega\left(\beta_{Y}(C)\right),
$$

where $\beta_{X}$ is the Hausdorff measure of noncompactness on $X$ and $\beta_{Y}$ is the Hausdorff measure of noncompactness on $Y$.

A multi-function $F: D \leadsto X$ is called $\beta$-compact if it is u.s.c. and, for each bounded subset $C$ in $D$, (5.1) is satisfied.

In order to simplify the notation, in all that follows, whenever any possibility of confusion will be ruled out by the context, we will denote both functions $\beta_{X}$ and $\beta_{Y}$ with the very same symbol, $\beta$.

Remark 5.1. One may easily verify that each locally compact multi-function is locally $\beta$-compact. Also, each $\beta$-compact multi-function is locally $\beta$-compact. Furthermore, if $D$ is locally compact and $F: D \leadsto X$ is u.s.c. with nonempty and compact values, then $F$ is locally compact and thus locally $\beta$-compact. Therefore, if $Y$ is finite dimensional and $D \subseteq Y$ is locally closed, each u.s.c. multi-function $F: D \leadsto X$ with compact values is locally $\beta$-compact. Moreover, if $Y$ is finite dimensional and $D \subseteq Y$ is closed, each u.s.c. multi-function $F: D \leadsto X$ with compact values is $\beta$-compact.

Remark 5.2. Each locally $\beta$-compact multi-function has relatively compact values, because $\beta(F(\xi)) \leq \omega(\beta(\{\xi\}))=\omega(0)=0$ for each $\xi \in D$.

Theorem 5.1. Let $X$ be a Banach space, let $K \subseteq X$ be a nonempty and locally closed set and let $F: K \leadsto X$ be a locally $\beta$-compact multi-function with nonempty, closed and convex values. A necessary and sufficient condition in order that $K$ be exact viable with respect to $F$ is that

$$
F(\xi) \in \mathcal{T S}_{K}(\xi)
$$

for each $\xi \in K$.

From Theorem 5.1] Remark 5.2 and Proposition 3.5 we get:

Theorem 5.2. Let $X$ be a Banach space, let $K \subseteq X$ be a nonempty and locally closed set and let $F: K \leadsto X$ be a locally $\beta$-compact multi-function with nonempty, closed and convex values. A necessary and sufficient condition in order that $K$ be exact viable with respect to $F$ is that

$$
F(\xi) \cap \mathcal{T}_{K}(\xi) \neq \emptyset
$$

for each $\xi \in K$. 
From Theorem 5.2 and Remark 5.1, we deduce

Theorem 5.3. Let $X$ be finite dimensional, $K \subseteq X$ a nonempty and locally closed set and let $F: K \leadsto X$ be a u.s.c. multi-function with nonempty, compact and convex values. A necessary and sufficient condition in order that $K$ be exact viable with respect to $F$ is the tangency condition (5.3).

A result of a different topological nature is

Theorem 5.4. Let $X$ be a Banach space, let $K \subseteq X$ be a nonempty and locally compact set and let $F: K \leadsto X$ be a strongly-weakly u.s.c. multi-function with nonempty, weakly compact and convex values. Then, a sufficient condition in order that $K$ be exact viable with respect to $F$ is the tangency condition (5.2).

Since in reflexive Banach spaces the class of weakly relatively compact subsets coincides with the one of bounded subsets, from Theorem 5.4, we deduce

Corollary 5.1. Let $X$ be a reflexive Banach space, let $K \subseteq X$ be a nonempty and locally compact set and let $F: K \leadsto X$ be a strongly-weakly u.s.c. multi-function with nonempty, bounded, closed and convex values. Then, a sufficient condition in order that $K$ be exact viable with respect to $F$ is the tangency condition (5.2).

Remark 5.3. In the hypotheses of Theorem 5.4, if the function $u:[0, T] \rightarrow K$ is an exact solution of (4.1), by Corollary 4.2, we conclude that whenever $F(u(t)) \in$ $\mathcal{T S}_{K}(u(t))$ but $F(u(t)) \cap \mathcal{T}_{K}(u(t))=\emptyset$, then the ratio $h \mapsto h^{-1}(u(t+h)-u(t))$ has no limit points as $h \downarrow 0$. So, if an exact solution reaches a point $x \in K$ with $F(x) \in \mathcal{T S}_{K}(x)$ and $F(x) \cap \mathcal{T}_{K}(x)=\emptyset$, it crosses $x$ along a completely nonsmooth (at that point) trajectory.

The main goal of the next two sections is to prove Theorems 5.1 and 5.4 As the necessity part follows from Theorem 4.2 combined with Remark 5.2, we will focus our attention only on the sufficiency part.

\section{Existence of $\varepsilon$-Approximate exaCt solutions}

The first step in the proof of the sufficiency is concerned with the existence of "approximate solutions" to the autonomous Cauchy problem for the differential inclusion

$$
\left\{\begin{array}{l}
u^{\prime}(t) \in F(u(t)) \\
u(0)=\xi
\end{array}\right.
$$

where $K \subseteq X$ is locally closed, $\xi \in K$ and $F: K \leadsto X$ is locally bounded. Since $K$ is locally closed, there exists $\rho>0$ such that the set $D(\xi, \rho) \cap K$ is closed. Next, taking into account that $F$ is bounded on $D(\xi, \rho) \cap K$ provided $\rho$ is small enough, diminishing $\rho>0$ if necessary, we can choose $M>0$ and $T>0$ such that

$$
\|y\| \leq M
$$

for every $x \in D(\xi, \rho) \cap K$ and $y \in F(x)$, and

$$
T(M+1) \leq \rho .
$$

Lemma 6.1. Let $X$ be a Banach space, let $K \subseteq X$ be nonempty and locally closed and let $F: K \leadsto X$ be locally bounded and satisfying $F(\xi) \in \mathcal{T S}_{K}(\xi)$ for each $\xi \in K$. Let $\xi \in K, \rho>0, M>0$ and $T>0$ be fixed as above. Then, for each $\varepsilon \in(0,1)$, 
there exist $\sigma:[0, T] \rightarrow[0, T]$ nondecreasing, $f:[0, T] \rightarrow X$ and $g:[0, T] \rightarrow X$ Bochner integrable and $u:[0, T] \rightarrow X$ continuous, such that:

(i) $t-\varepsilon \leq \sigma(t) \leq t$ for each $t \in[0, T]$;

(ii) $\|g(t)\| \leq \varepsilon$ a.e. for $t \in[0, T]$;

(iii) $u(\sigma(t)) \in D(\xi, \rho) \cap K$ for all $t \in[0, T]$ and $u(T) \in D(\xi, \rho) \cap K$;

(iv) $f(s) \in F(u(\sigma(s)))$ a.e. for $s \in[0, T]$;

(v) $u(t)=\xi+\int_{0}^{t} f(s) d s+\int_{0}^{t} g(s) d s$ for each $t \in[0, T]$.

Definition 6.1. A quadruple $(\sigma, f, g, u)$, satisfying (i) (v) in Lemma 6.1, is called an $\varepsilon$-approximate exact solution to the Cauchy problem (6.1) on the interval $[0, T]$.

We may now pass to the proof of Lemma 6.1

Proof. Let $\varepsilon>0$ be arbitrary. We begin by showing the existence of an $\varepsilon$ approximate exact solution on an interval $[0, \delta]$ with $\delta \in(0, T]$. By hypothesis, $F(\xi) \in \mathcal{T S}_{K}(\xi)$. From the equivalence between (i) and (iv) in Proposition [3.2, it follows that there exist $\eta \in F(\xi), \delta \in(0, T], \delta \leq \varepsilon$ and $p \in X$ with $\|p\| \leq \varepsilon$, such that

$$
\xi+\delta \eta+\delta p \in K
$$

Let $\sigma:[0, \delta] \rightarrow[0, \delta], f:[0, \delta] \rightarrow X, g:[0, \delta] \rightarrow X$ and $u:[0, \delta] \rightarrow X$ be defined by

$$
\begin{cases}\sigma(t)=0 & \text { for } t \in[0, \delta] \\ f(t)=\eta & \text { for } t \in[0, \delta] \\ g(t)=p & \text { for } t \in[0, \delta] \\ u(t)=\xi+t \eta+t p & \text { for } t \in[0, \delta] .\end{cases}
$$

One can readily see that the quadruple $(\sigma, f, g, u)$ is an $\varepsilon$-approximate exact solution to the Cauchy problem (6.1) on the interval $[0, \delta]$.

Next, we will prove the existence of an $\varepsilon$-approximate exact solution defined on the whole interval $[0, T]$. To this aim we shall make use of the Brezis-Browder Theorem 2.1, as follows. Let $\mathcal{S}$ be the set of all $\varepsilon$-approximate exact solutions to the problem (6.1) having the domains of definition of the form $[0, c]$ with $c \in(0, T]$. On $\mathcal{S}$ we define the relation " $\preceq$ " by

$$
\left(\sigma_{1}, f_{1}, g_{1}, u_{1}\right) \preceq\left(\sigma_{2}, f_{2}, g_{2}, u_{2}\right)
$$

if the domain of definition $\left[0, c_{1}\right]$ of the first quadruple is included in the domain of definition $\left[0, c_{2}\right]$ of the second quadruple and the two $\varepsilon$-approximate exact solutions coincide on the common part of the domains. Obviously " $\preceq$ " is a preorder relation on $\mathcal{S}$. Let us show first that each increasing sequence $\left(\left(\sigma_{m}, f_{m}, g_{m}, u_{m}\right)\right)_{m}$ is bounded from above. Indeed, let $\left(\left(\sigma_{m}, f_{m}, g_{m}, u_{m}\right)\right)_{m}$ be an increasing sequence, and let

$$
c^{*}=\lim _{m} c_{m},
$$

where $\left[0, c_{m}\right]$ denotes the domain of definition of $\left(\sigma_{m}, f_{m}, g_{m}, u_{m}\right)$. We will show that there exists at least one element, $\left(\sigma^{*}, f^{*}, g^{*}, u^{*}\right) \in \mathcal{S}$, defined on $\left[0, c^{*}\right]$ and satisfying $\left(\sigma_{m}, f_{m}, g_{m}, u_{m}\right) \preceq\left(\sigma^{*}, f^{*}, g^{*}, u^{*}\right)$ for each $m \in \mathbb{N}$. In order to do this, we have to prove first that there exists $\lim _{m} u_{m}\left(c_{m}\right)$. For each $m, k \in \mathbb{N}, m \leq k$, 
we have $u_{m}(s)=u_{k}(s)$ for all $s \in\left[0, c_{m}\right]$. Taking into account (iii), (iv), (v) and (6.2), we deduce

$$
\left\|u_{m}\left(c_{m}\right)-u_{k}\left(c_{k}\right)\right\| \leq \int_{c_{m}}^{c_{k}}\left[\left\|f_{k}(\theta)\right\|+\left\|g_{k}(\theta)\right\|\right] d \theta \leq(M+\varepsilon)\left|c_{k}-c_{m}\right|
$$

for every $m, k \in \mathbb{N}$, which proves that there exists $\lim _{m} u_{m}\left(c_{m}\right)$. Since for every $m \in \mathbb{N}, u_{m}\left(c_{m}\right) \in D(\xi, \rho) \cap K$, and the latter is closed, it readily follows that $\lim _{m} u_{m}\left(c_{m}\right) \in D(\xi, \rho) \cap K$. Furthermore, because all the functions in the set $\left\{\sigma_{m} ; m \in \mathbb{N}\right\}$ are nondecreasing, with values in $\left[0, c^{*}\right]$, and satisfy $\sigma_{m}\left(c_{m}\right) \leq \sigma_{p}\left(c_{p}\right)$ for every $m, p \in \mathbb{N}$ with $m \leq p$, there exists $\lim _{m} \sigma_{m}\left(c_{m}\right)$ and this limit belongs to $\left[0, c^{*}\right]$. So, we can define $\left(\sigma^{*}, f^{*}, g^{*}, u^{*}\right):\left[0, c^{*}\right] \rightarrow\left[0, c^{*}\right] \times X \times X \times X$ by

$$
\begin{aligned}
& \sigma^{*}(t)= \begin{cases}\sigma_{m}(t) & \text { for } t \in\left[0, c_{m}\right] \\
\lim _{m} \sigma_{m}\left(c_{m}\right) & \text { for } t=c^{*},\end{cases} \\
& u^{*}(t)= \begin{cases}u_{m}(t) & \text { for } t \in\left[0, c_{m}\right] \\
\lim _{m} u_{m}\left(c_{m}\right) & \text { for } t=c^{*},\end{cases} \\
& g^{*}(t)= \begin{cases}g_{m}(t) & \text { for } t \in\left[0, c_{m}\right] \\
0 & \text { for } t=c^{*},\end{cases} \\
& f^{*}(t)= \begin{cases}f_{m}(t) & \text { for } t \in\left[0, c_{m}\right] \\
\eta^{*} & \text { for } t=c^{*},\end{cases}
\end{aligned}
$$

where $\eta^{*}$ is an arbitrary but fixed element in $F\left(u^{*}\left(\sigma^{*}\left(c^{*}\right)\right)\right)$. One can easily see that $\left(\sigma^{*}, f^{*}, g^{*}, u^{*}\right)$ is an $\varepsilon$-approximate exact solution which is an upper bound for $\left(\left(\sigma_{m}, f_{m}, g_{m}, u_{m}\right)\right)_{m}$. Let us define the function $\mathcal{N}: \mathcal{S} \rightarrow \mathbb{R} \cup\{+\infty\}$ by $\mathcal{N}((\sigma, f, g, u))=c$, where $[0, c]$ is the domain of definition of $(\sigma, f, g, u)$. Clearly $\mathcal{N}$ satisfies the hypotheses of the Brezis-Browder Theorem 2.1. Then, $\mathcal{S}$ contains at least one $\mathcal{N}$-maximal element $(\bar{\sigma}, \bar{f}, \bar{g}, \bar{u})$, defined on $[0, \bar{c}]$. In other words, if $(\widetilde{\sigma}, \widetilde{f}, \widetilde{g}, \widetilde{u}) \in \mathcal{S}$, defined on $[0, \widetilde{c}]$, satisfies $(\bar{\sigma}, \bar{f}, \bar{g}, \bar{u}) \preceq(\widetilde{\sigma}, \widetilde{f}, \widetilde{g}, \widetilde{u})$, then we necessarily have $\bar{c}=\widetilde{c}$. We will next show that $\bar{c}=T$. Indeed, let us assume by contradiction that $\bar{c}<T$. Since

$$
\begin{gathered}
\|\bar{u}(\bar{c})-\xi\| \leq \int_{0}^{\bar{c}}\|\bar{f}(s)\| d s+\int_{0}^{\bar{c}}\|\bar{g}(s)\| d s \leq \bar{c}(M+\varepsilon) \\
\leq \bar{c}(M+1)<T(M+1),
\end{gathered}
$$

we deduce that

$$
\|\bar{u}(\bar{c})-\xi\|<\rho .
$$

Then, as $\bar{u}(\bar{c}) \in K$, we have $F(\bar{u}(\bar{c})) \in \mathcal{T S}_{K}(\bar{u}(\bar{c}))$ and thus, again by the equivalence between (i) and (iv) in Proposition 3.2, there exist $\bar{\eta} \in F(\bar{u}(\bar{c})), \delta \in(0, T-\bar{c}), \delta \leq \varepsilon$ and $p \in X,\|p\| \leq \varepsilon$, such that $\bar{u}(\bar{c})+\delta \bar{\eta}+\delta p \in K$. From (6.4), it follows that we can diminish $\delta$ if necessary 2 in order to have

$$
\|\bar{u}(\bar{c})+\delta \bar{\eta}+\delta p-\xi\| \leq \rho .
$$

\footnotetext{
${ }^{2}$ Of course, both $\bar{\eta}$ and $p$ may change with $\delta$.
} 
Let us define the functions $\sigma:[0, \bar{c}+\delta] \rightarrow[0, \bar{c}+\delta], f:[0, \bar{c}+\delta] \rightarrow X$ and $g:[0, \bar{c}+\delta] \rightarrow X$ by

$$
\begin{aligned}
& \sigma(t)= \begin{cases}\bar{\sigma}(t) & \text { for } t \in[0, \bar{c}] \\
\bar{c} & \text { for } t \in(\bar{c}, \bar{c}+\delta],\end{cases} \\
& f(t)=\left\{\begin{array}{ll}
\bar{f}(t) & \text { for } t \in[0, \bar{c}] \\
\bar{\eta} & \text { for } t \in(\bar{c}, \bar{c}+\delta],
\end{array} \quad g(t)= \begin{cases}\bar{g}(t) & \text { for } t \in[0, \bar{c}] \\
p & \text { for } t \in(\bar{c}, \bar{c}+\delta] .\end{cases} \right.
\end{aligned}
$$

Clearly, $f$ and $g$ are Bochner integrable on $[0, \bar{c}+\delta]$ and $\|g(t)\| \leq \varepsilon$ for every $t \in[0, \bar{c}+\delta]$. We define $u:[0, \bar{c}+\delta] \rightarrow X$ by

$$
u(t)= \begin{cases}\bar{u}(t) & \text { for } t \in[0, \bar{c}] \\ \bar{u}(\bar{c})+(t-\bar{c}) \bar{\eta}+(t-\bar{c}) p & \text { for } t \in(\bar{c}, \bar{c}+\delta] .\end{cases}
$$

We notice that

$$
u(t)=\xi+\int_{0}^{t} f(\theta) d \theta+\int_{0}^{t} g(\theta) d \theta
$$

for every $t \in[0, \bar{c}+\delta]$. Thus $\sigma, f, g$ and $u$ satisfy the conditions (i), (ii), (iv) and (v). Since

$$
u(\sigma(t))= \begin{cases}\bar{u}(\bar{\sigma}(t)) & \text { for } t \in[0, \bar{c}] \\ \bar{u}(\bar{c}) & \text { for } t \in(\bar{c}, \bar{c}+\delta],\end{cases}
$$

it follows that $u(\sigma(t)) \in D(\xi, \rho) \cap K$ and thus (iii) is also satisfied. Furthermore, from the choice of $\delta$ and $p$, we have $u(\bar{c}+\delta)=\bar{u}(\bar{c})+\delta \bar{\eta}+\delta p \in K$. Moreover, from (6.5), we conclude $\|u(\bar{c}+\delta)-\xi\|=\|\bar{u}(\bar{c})+\delta \bar{\eta}+\delta p-\xi\| \leq \rho$ and consequently $u$ satisfies (iii). Thus $(\sigma, f, g, u) \in \mathcal{S}$.

Finally, inasmuch as $(\bar{\sigma}, \bar{f}, \bar{g}, \bar{u}) \preceq(\sigma, f, g, u)$ and $\bar{c}<\bar{c}+\delta$, it follows that $(\bar{\sigma}, \bar{f}, \bar{g}, \bar{u})$ is not an $\mathcal{N}$-maximal element. But this is absurd. This contradiction can be eliminated only if each maximal element in the set $\mathcal{S}$ is defined on $[0, T]$.

\section{Convergence of $\varepsilon$-Approximate exact solutions}

The goal of this section is to prove both Theorems 5.1 and 5.4. We will do that by showing the convergence of a suitably chosen sequence of $\varepsilon$-approximate exact solutions. Let us consider a sequence $\left(\varepsilon_{n}\right)_{n}$ in $(0,1)$, decreasing to 0 , and let $\left(\left(\sigma_{n}, f_{n}, g_{n}, u_{n}\right)\right)_{n}$ be a sequence of $\varepsilon_{n}$-approximate solutions of (6.1) on [0,T]. Let us observe that, by (i), (ii), (iv) and (v) 3 we have

$$
\left\|u_{n}\left(\sigma_{n}(t)\right)-u_{n}(t)\right\| \leq(M+1) \varepsilon_{n}
$$

for each $t \in[0, T]$.

We begin with the proof of Theorem 5.1

Proof. Let $M>0$ as in (6.2). Diminishing $\rho>0$, if necessary, we may assume that $F$ is $\beta$-compact on $D(\xi, \rho) \cap K$.

We analyze first the case when $X$ is separable. To begin with, let us observe that, in view of (7.1), $\left\{u_{n}(t)-u_{n}\left(\sigma_{n}(t)\right) ; n \in \mathbb{N}\right\}$ is relatively compact and so, for each $k \in \mathbb{N}, \beta\left(\left\{u_{n}\left(\sigma_{n}(t)\right)-u_{n}(t) ; n \geq k\right\}\right)=0$. Similarly, by (ii), it follows that, for each $k \in \mathbb{N}, \beta\left(\left\{\int_{0}^{t} g_{n}(s) d s ; n \geq k\right\}\right)=0$. From these remarks, (7.1), (v), the

\footnotetext{
${ }^{3}$ Within this section, all the quotations to items like $(i) \sim(v)$ refer to the corresponding items in Lemma 6.1
} 
fact that $F$ is $\beta$-compact on $D(\xi, \rho) \cap K$, Lemma 2.3 and Proposition 2.3. it follows that

$$
\begin{gathered}
\beta\left(\left\{u_{n}(t) ; n \geq k\right\}\right) \leq \beta\left(\left\{\int_{0}^{t} f_{n}(s) d s ; n \geq k\right\}\right)+\beta\left(\left\{\int_{0}^{t} g_{n}(s) d s ; n \geq k\right\}\right) \\
\leq \int_{0}^{t} \beta\left(\left\{f_{n}(s) ; n \geq k\right\}\right) d s \leq \int_{0}^{t} \omega\left(\beta\left(\left\{u_{n}\left(\sigma_{n}(s)\right) ; n \geq k\right\}\right)\right) d s \\
\leq \int_{0}^{t} \omega\left(\beta\left(\left\{u_{n}(s) ; n \geq k\right\}+\left\{u_{n}\left(\sigma_{n}(s)\right)-u_{n}(s) ; n \geq k\right\}\right)\right) d s \\
\leq \int_{0}^{t} \omega\left(\beta\left(\left\{u_{n}(s) ; n \geq k\right\}\right)+\beta\left(\left\{u_{n}\left(\sigma_{n}(s)\right)-u_{n}(s) ; n \geq k\right\}\right)\right) d s \\
=\int_{0}^{t} \omega\left(\beta\left(\left\{u_{n}(s) ; n \geq k\right\}\right)\right) d s .
\end{gathered}
$$

Set $x_{k}(t)=\beta\left(\left\{u_{n}(t) ; n \geq k\right\}\right)$. The inequality above can be rewriten as

$$
x_{k}(t) \leq \int_{0}^{t} \omega\left(x_{k}(s)\right) d s
$$

for each $t \in[0, T]$. By Lemma 2.5 diminishing $T>0$, if necessary, we may assume that $\lim _{k} x_{k}(t)=0$ uniformly for $t \in[0, T]$. Then $\lim _{k} \beta\left(\left\{u_{n}(s) ; n \geq k\right\}\right)=0$, and thus we are in the hypotheses of Lemma 2.4. It follows that, for each $t \in[0, T]$, $\left\{u_{n}(t) ; n=1,2, \ldots\right\}$ is relatively compact. By (v) and (6.2) we conclude that $\left\{u_{n} ; n=1,2, \ldots\right\}$ is equicontinuous, and therefore, thanks to the Arzelà-Ascoli Theorem 2.7, there exists $u \in C([0, T] ; X)$ such that, on a subsequence at least,

$$
\lim _{n} u_{n}(t)=u(t)
$$

uniformly for $t \in[0, T]$. In view of (7.1), we also have

$$
\lim _{n} u_{n}\left(\sigma_{n}(t)\right)=u(t)
$$

uniformly for $t \in[0, T]$. From (iii) and the fact that $D(\xi, \rho) \cap K$ is closed, we deduce that $u(t) \in D(\xi, \rho) \cap K$ for each $t \in[0, T]$.

In view of Remark 5.2, $F$ has compact and thus weakly compact values. We shall apply Theorem 2.6 to show that $u$ is both absolutely continuous and a.e. differentiable on $[0, T]$, and

$$
u(t)=\xi+\int_{0}^{t} u^{\prime}(s) d s
$$

for each $t \in[0, T]$. Indeed, since $f_{n}(s) \in F\left(u_{n}\left(\sigma_{n}(s)\right)\right)$ for $n=1,2, \ldots$ and $s \in$ $[0, T]$, and, by (6.2), $F(D(\xi, \rho) \cap K)$ is bounded, it follows that $\left\{f_{n} ; n=1,2, \ldots\right\}$ is uniformly integrable. Moreover, as $F$ is u.s.c. and has weakly compact values, in view of Lemma 2.1.

$$
\left\{F\left(u_{n}\left(\sigma_{n}(t)\right)\right) ; n=1,2, \ldots, t \in[0, T]\right\}
$$

is weakly compact. So, by Theorem 2.3 , its closed convex hull is weakly compact too and thus we are in the hypotheses of Theorem 2.6. which, along with Theorem 2.4. shows that $\left(f_{n}\right)_{n}$ has at least one weakly convergent subsequence in $L^{1}(0, T ; X)$ to some function $f$. Summarizing, we have $\lim _{n} u_{n}=u$ uniformly on $[0, T]$ and 
$\lim _{n} u_{n}^{\prime}=f$ weakly in $L^{1}(0, T ; X)$. Thus $u$ is absolutely continuous and a.e. differentiable on $[0, T], u^{\prime}(t)=f(t)$ a.e. for $t \in[0, T]$ and $u$ is a primitive of its derivative $u^{\prime}$.

It remains to be shown that, at each differentiability point, $t \in[0, T]$, of $u$, we have $u^{\prime}(t) \in F(u(t))$. Let $t \in[0, T)$ be a differentiability point of $u$ and let $h>0$ be such that $t+h \in[0, T]$. Let $n=1,2, \ldots$ be arbitrary but fixed. We have

$$
\frac{1}{h}\left(u_{n}(t+h)-u_{n}(t)\right)=\frac{1}{h} \int_{t}^{t+h} f_{n}(s) d s+\frac{1}{h} \int_{t}^{t+h} g_{n}(s) d s
$$

where $f_{n}(s) \in F\left(u_{n}\left(\sigma_{n}(s)\right)\right)$ for each $s \in[t, t+h]$. Let $\varepsilon>0$. Since $F$ is u.s.c. at $u(t), u$ is continuous, $\lim _{n} \sigma_{n}(s)=s$ and $\lim _{n} u_{n}\left(\sigma_{n}(s)\right)=u(s)$, uniformly for $s \in[0, T]$, there exists $h(\varepsilon)>0$ and $n(\varepsilon) \in \mathbb{N}$ such that, for each $h \in(0, h(\varepsilon)]$ and each $n \geq n(\varepsilon)$, we have

$$
F\left(u_{n}\left(\sigma_{n}(s)\right)\right) \subseteq F(u(t))+D(0, \varepsilon)
$$

for each $s \in[t, t+h]$ and

$$
\left\|\frac{1}{h} \int_{t}^{t+h} g_{n}(s) d s\right\| \leq \varepsilon .
$$

Since

$$
\frac{1}{h} \int_{t}^{t+h} f_{n}(s) d s \in \overline{\mathrm{conv}} \bigcup_{s \in[t, t+h]} F\left(u_{n}\left(\sigma_{n}(s)\right)\right)
$$

and $F(u(t))+D(0, \varepsilon)$ is convex because both $F(u(t))$ and $D(0, \varepsilon)$ are convex, it follows that

$$
\frac{1}{h}\left(u_{n}(t+h)-u_{n}(t)\right) \in F(u(t))+D(0,2 \varepsilon)
$$

for each $h \in(0, h(\varepsilon)]$ and each $n \geq n(\varepsilon)$. Keeping $h$ fixed in $(0, h(\varepsilon)]$, passing to the limit for $n \rightarrow \infty$ in this relation and taking into account that $F(u(t))+D(0,2 \varepsilon)$ is closed (because $F(u(t))$ is compact), we get

$$
\frac{1}{h}(u(t+h)-u(t)) \in F(u(t))+D(0,2 \varepsilon) .
$$

Finally, passing to the limit for $h \downarrow 0$ in this relation we get $u^{\prime}(t) \in F(u(t))$. Since the case $t=T$ can be treated similarly by computing the left derivative of $u$ at $T$, the proof of Theorem [5.1] is complete in the case when $X$ is separable.

If $X$ is not separable, there exists a separable and closed subspace, $Y$, of $X$ such that $u_{n}(t), f_{n}(t), g_{n}(t) \in Y$ for $n=1,2, \ldots$ and a.e. for $t \in[0, T]$. On the other hand, from Proposition 2.2. Definition 5.2 and the monotonicity of $\omega$, we deduce

$$
\beta_{Y}(F(C)) \leq 2 \beta(F(C)) \leq 2 \omega(\beta(C)) \leq 2 \omega\left(\beta_{Y}(C)\right),
$$

for each set $C \subseteq D(\xi, \rho) \cap K \cap Y$.

In view of Remark 2.2, $2 \omega$ is a uniqueness function too. Repeating the routine above, with $\beta$ replaced by $\beta_{Y}$ and $\omega$ replaced by $2 \omega$, using Remark 2.1 instead of Lemma 2.3 and the fact that the restriction of $\beta_{Y}$ (as defined in Proposition 2.2) to 
$\mathcal{B}(Y)$ is the Hausdorff measure of noncompactness on $Y$, we conclude that, for each $t \in[0, T],\left\{u_{n}(t) ; n=1,2, \ldots\right\}$ is relatively compact in $Y$. But $Y$ is a subspace of $X$ and, from now on, we have to repeat the same arguments as in the case when $X$ is separable. This completes the proof.

We can now proceed to the proof of Theorem 5.4.

Proof. Let $\left(\varepsilon_{n}\right)_{n}$ be a sequence in $(0,1)$, decreasing to 0 , and let $\left(\left(\sigma_{n}, f_{n}, g_{n}, u_{n}\right)\right)_{n}$ be a sequence of $\varepsilon_{n}$-approximate solutions of (6.1) on $[0, T]$. Since $K$ is locally compact and $F$ is strongly-weakly u.s.c. and has nonempty and weakly compact values, by Lemma 2.1, it follows that $F$ is locally bounded. Then, we can find $M>0$ satisfying (6.2). Diminishing $\rho>0$ and $T>0$ if necessary, we may assume that the conclusion of Lemma 6.1 holds true and, in addition, $D(\xi, \rho) \cap K$ is compact. Taking into account that, by (iii), $u_{n}\left(\sigma_{n}(t)\right) \in D(\xi, \rho) \cap K$ for $n=1,2, \ldots$ and $t \in[0, T]$, it follows that, for each $t \in[0, T],\left\{u_{n}\left(\sigma_{n}(t)\right) ; n=1,2, \ldots\right\}$ is relatively compact in $X$. In view of (17.1), we conclude that, for each $t \in[0, T],\left\{u_{n}(t) ; n=1,2, \ldots\right\}$ is relatively compact in $X$, too.

From (6.2) and (ii) and (v), we deduce that $\left\{u_{n} ; n=1,2, \ldots\right\}$ is equicontinuous on $[0, T]$. By the Arzelà-Ascoli Theorem 2.7, we conclude that there exists $u \in C([0, T] ; X)$ such that, on a subsequence at least, we have $\lim _{n} u_{n}(t)=u(t)$ uniformly for $t \in[0, T]$. In view of this relation, of (i) and (iii), we deduce that $\lim _{n} u_{n}\left(\sigma_{n}(t)\right)=u(t)$ uniformly for $t \in[0, T]$ and $u(t) \in D(\xi, \rho) \cap K$.

As $D(\xi, \rho) \cap K$ is compact and $F$ is strongly-weakly u.s.c. and has convex and weakly compact values, by Lemma 2.1, it follows that the set $F(D(\xi, \rho) \cap K)$ is bounded, being weakly relatively compact. Then, as $f_{n}(s) \in F\left(u_{n}\left(\sigma_{n}(s)\right)\right)$ for $n=$ $1,2, \ldots$ and $s \in[0, T]$, it follows that $\left\{f_{n} ; n=1,2, \ldots\right\}$ is uniformly integrable. By Theorem 2.6 combined with Theorem 2.4, we deduce that $\left(f_{n}\right)_{n}$ has at least one weakly convergent subsequence in $L^{1}(0, T ; X)$ to some function $f$. Summarizing, we have $\lim _{n} u_{n}=u$ uniformly on $[0, T]$ and $\lim _{n} u_{n}^{\prime}=f$ weakly in $L^{1}(0, T ; X)$. Thus $u$ is absolutely continuous, a.e. differentiable on $[0, T], u^{\prime}(t)=f(t)$ a.e. for $t \in[0, T]$ and $u$ is a primitive of its derivative $u^{\prime}$. From (iv) and Lemma 2.2, we conclude that $f(t) \in F(u(t))$ a.e. for $t \in[0, T]$. Thus $u$ is an almost exact solution of (4.1) on $[0, T]$. As $\xi \in K$ is arbitrary, this shows that $K$ is almost exact viable with respect to $F$. To complete the proof, it remains to show that $u$ is even an exact solution of (4.1) on $[0, T]$. To this aim, let $t \in[0, T)$ be a differentiability point of $u$ and let $E$ be an arbitrary open half-space with $F(u(t)) \subseteq E$. Since $E$ is weakly open too, $u$ is continuous and $F$ is strongly-weakly u.s.c at $u(t)$, there exists $\delta(E)>0$ such that, for each $h \in(0, \delta(E)]$, with $t+h \leq T$, we have

$$
F(u(s)) \subseteq E
$$

for each $s \in[t, t+h]$. Consequently, for $h$ as above, we have

$$
\bigcup_{s \in[t, t+h]} F(u(s)) \subseteq E .
$$

On the other hand, for $n=1,2, \ldots$,

$$
\frac{1}{h}\left(u_{n}(t+h)-u_{n}(t)\right)=\frac{1}{h} \int_{t}^{t+h} f_{n}(s) d s+\frac{1}{h} \int_{t}^{t+h} g_{n}(s) d s .
$$


Since $\lim _{n} u_{n}\left(\sigma_{n}(s)\right)=u(s)$ uniformly on $[0, T]$ and $f_{n}(s) \in F\left(u_{n}\left(\sigma_{n}(s)\right)\right)$ for each $s \in[0, T]$, there exists $n(E)=1,2, \ldots$ such that, for all $n \geq n(E)$,

$$
\frac{1}{h} \int_{t}^{t+h} f_{n}(s) d s \in \overline{\mathrm{conv}} \bigcup_{s \in[t, t+h]} F\left(u_{n}\left(\sigma_{n}(s)\right)\right) \subseteq \bar{E} .
$$

Therefore

$$
\frac{1}{h}\left(u_{n}(t+h)-u_{n}(t)\right)-\frac{1}{h} \int_{t}^{t+h} g_{n}(s) d s \in \bar{E} .
$$

Recalling that $\lim _{n}\left\|g_{n}(s)\right\|=0$ uniformly for $s \in[0, T]$ and passing to the limit successively for $n \rightarrow \infty$ and $h \downarrow 0$ in the last relation, we get $u^{\prime}(t) \in \bar{E}$. Since $E$ is an arbitrary open half-space including $F(u(t))$ and the latter, being convex and closed, is the intersection of all closed half-spaces including it, we conclude that $u^{\prime}(t) \in F(u(t))$. Since the case $t=T$ can be handled similarly, this completes the proof.

\section{The nonautonomous U.S.C. Case}

In this section we will show how all the results established before for the autonomous differential inclusion $u^{\prime}(t) \in F(u(t))$ extend to the nonautonomous one $u^{\prime}(t) \in F(t, u(t))$. So, let $X$ be a real Banach space, $\mathcal{C}$ a nonempty subset in $\mathbb{R} \times X$, $F: \mathcal{C} \leadsto X$ a given multi-function, $(\tau, \xi) \in \mathcal{C}$ and let us consider the Cauchy problem for the nonautonomous differential inclusion

$$
\left\{\begin{array}{l}
u^{\prime}(t) \in F(t, u(t)) \\
u(\tau)=\xi
\end{array}\right.
$$

Definition 8.1. By an exact solution of (8.1) on $[\tau, T]$ we mean an absolutely continuous function $u:[\tau, T] \rightarrow X$, a.e. differentiable on $[\tau, T]$, with $u^{\prime} \in L^{1}(\tau, T ; X)$, and satisfying:

(i) $(t, u(t)) \in \mathcal{C}$ for each $t \in[\tau, T]$ and

(ii) $u^{\prime}(t) \in F(t, u(t))$ at every point $t \in[\tau, T]$ at which $u$ is differentiable, and $u(\tau)=\xi$

An exact solution of (8.1) on the semi-open interval $[\tau, T)$ is defined similarly.

Definition 8.2. By an almost exact solution of (8.1) on $[\tau, T]$ we mean an absolutely continuous function $u:[\tau, T] \rightarrow X$, a.e. differentiable on $[\tau, T]$, with $u^{\prime} \in L^{1}(\tau, T ; X)$, and satisfying:

(i) $(t, u(t)) \in \mathcal{C}$ for each $t \in[\tau, T]$ and

(ii) $u^{\prime}(t) \in F(t, u(t))$ a.e. for $t \in[\tau, T]$, and $u(\tau)=\xi$.

An almost exact solution of (8.1) on the semi-open interval $[\tau, T)$ is defined similarly, noticing that, in this case, we have to impose the weaker constraint $u^{\prime} \in$ $L_{\text {loc }}^{1}([\tau, T) ; X)$.

Definition 8.3. The set $\mathcal{C}$ is exact viable (almost exact viable) with respect to $F$ if for each $(\tau, \xi) \in \mathcal{C}$ there exist $T \in \mathbb{R}, T>\tau$, and a exact solution (almost exact solution) $u:[\tau, T] \rightarrow X$ of (8.1).

We will rewrite the nonautonomous problem above as an autonomous one in the space $X=\mathbb{R} \times X$, endowed with the norm $\|(t, u)\|=|t|+\|u\|$, for each $(t, u) \in X$. 
Namely, set $z(s)=(t(s), u(s))$ and $\mathcal{F}(z)=(1, F(z))$, for $s \in[0, T-\tau]$, where $(1, F(z))=\{(1, y) ; y \in F(z)\}$. Then, the Cauchy problem above is equivalent to

$$
\left\{\begin{array}{l}
z^{\prime}(s) \in \mathcal{F}(z(s)) \\
z(0)=(\tau, \xi)
\end{array}\right.
$$

So, all the viability results proved before extend in an obvious way to the nonautonomous case via the transformations above. Namely, we have

Theorem 8.1. If $\mathcal{C}$ is almost exact viable with respect to $F: \mathcal{C} \leadsto X$, then, at each point $(\tau, \xi) \in \mathcal{C}$ at which $F$ is u.s.c. and $F(\tau, \xi)$ is convex, we have $(1, F(\tau, \xi)) \in$ $\mathcal{T} \mathcal{S}_{\mathcal{C}}(\tau, \xi)$.

Remark 8.1. If $\mathcal{C}$ is a cylindrical domain, i.e. $\mathcal{C}=I \times K$ with $I$ an open to the right interval and $K$ a subset in $X$, then, for each $(\tau, \xi) \in \mathcal{C}$, the following two tangency conditions

(i) $(1, F(\tau, \xi)) \in \mathcal{T S}_{\mathcal{C}}(\tau, \xi)$,

(ii) $F(\tau, \xi) \in \mathcal{T S}_{K}(\xi)$

are equivalent.

Theorem 8.2. Let $X$ be a Banach space, $\mathcal{C} \subseteq \mathbb{R} \times X$ a nonempty and locally closed set and let $F: \mathcal{C} \leadsto X$ be a locally $\beta$-compact multi-function with nonempty, closed and convex values. A necessary and sufficient condition in order that $\mathcal{C}$ be exact viable with respect to $F$ is the tangency condition

$$
(1, F(\tau, \xi)) \in \mathcal{T S}_{\mathcal{C}}(\tau, \xi)
$$

for each $(\tau, \xi) \in \mathcal{C}$.

Proof. Let us observe that $u:[\tau, T] \rightarrow X$ is an exact solution of (8.1) if and only if $z:[0, T-\tau] \rightarrow \mathcal{C}, z(s)=(s+\tau, u(s+\tau))$ is an exact solution of the autonomous Cauchy problem (8.2). Since $F$ is u.s.c. and satisfies both $F(z) \in \mathcal{T} \mathcal{S}_{\mathcal{C}}(z)$ for each $z \in \mathcal{C}$ and

$$
\beta_{x}(\mathcal{F}(B))=\beta_{x}(\{1\} \times F(B))=\beta_{X}(F(B)) \leq \omega\left(\beta_{X}(B)\right)
$$

for each bounded subset $B$ in $\mathcal{C}$, the conclusion follows from Theorem 5.2 .

A corollary of Theorem 8.2, Remark [5.1 and Proposition 3.5] is

Theorem 8.3. Let $X$ be a Banach space, $\mathcal{C} \subseteq \mathbb{R} \times X$ a nonempty and locally closed set and let $F: \mathcal{C} \leadsto X$ be a locally $\beta$-compact multi-function with nonempty, closed and convex values. Then, a necessary and sufficient condition in order that $\mathcal{C}$ be exact viable with respect to $F$ is the tangency condition

$$
(1, F(\tau, \xi)) \cap \mathcal{T}_{\mathfrak{C}}(\tau, \xi) \neq \emptyset
$$

for each $(\tau, \xi) \in \mathcal{C}$.

The following proposition is an easy consequence of the definition of contingent cone.

Proposition 8.1. The pair $(1, y)$ belongs to $\mathcal{T}_{\mathfrak{C}}(\tau, \xi)$ if and only if there exist $\left(\tau_{n}, \xi_{n}\right) \in \mathcal{C}$ such that $\tau_{n} \downarrow \tau, \lim _{n} \xi_{n}=\xi$ and $\lim _{n} \frac{\xi_{n}-\xi}{\tau_{n}-\tau}=y$. 
From Theorem 8.3 we deduce

Theorem 8.4. Let $X$ be finite dimensional, let $\mathcal{C} \subseteq \mathbb{R} \times X$ be a nonempty and locally closed set and let $F: \mathcal{C} \leadsto X$ be a u.s.c. multi-function with nonempty, convex and compact values. Then, a necessary and sufficient condition in order that $\mathrm{C}$ be exact viable with respect to $F$ is the tangency condition (8.4).

A nonautonomous version of Theorem 5.4 is

Theorem 8.5. Let $X$ be a Banach space, $\mathcal{C} \subseteq \mathbb{R} \times X$ a nonempty and locally compact set and let $F: \mathcal{C} \leadsto X$ be a strongly-weakly u.s.c. multi-function with nonempty, weakly compact and convex values. Then, a sufficient condition in order that $\mathcal{C}$ be exact viable with respect to $F$ is the tangency condition (8.3).

\section{Global (almost) exact solutions}

Let $\mathcal{C} \subseteq \mathbb{R} \times X$ be nonempty and let $F: \mathcal{C} \leadsto X$. In this section we will prove some results concerning the existence of noncontinuable, or even global solutions to the Cauchy problem

$$
\left\{\begin{array}{l}
u^{\prime}(t) \in F(t, u(t)) \\
u(\tau)=\xi .
\end{array}\right.
$$

An (almost) exact solution $u:[\tau, T) \rightarrow X$ of (9.1) is called noncontinuable if there is no other (almost) exact solution $v:[\tau, \widetilde{T}) \rightarrow X$ of (9.1), with $T<\widetilde{T}$ and satisfying $u(t)=v(t)$ for all $t \in[\tau, T)$. The (almost) exact solution $u$ is called global if $T=T_{\mathcal{C}}$, with $T_{\mathcal{C}}$ given by

$$
T_{\mathcal{C}}=\sup \{t \in \mathbb{R} ; \text { there exists } \eta \in X \text {, with }(t, \eta) \in \mathcal{C}\} \text {. }
$$

The next theorem follows from the Brezis-Browder Theorem 2.1.

Theorem 9.1. Let $X$ be a Banach space, $\mathcal{C} \subseteq \mathbb{R} \times X$ be nonempty and $F: \mathcal{C} \leadsto X$. The following conditions are equivalent:

(i) $\mathcal{C}$ is (almost) exact viable with respect to $F$;

(ii) for each $(\tau, \xi) \in \mathcal{C}$ there exists at least one noncontinuable (almost) exact solution $u:[\tau, T) \rightarrow X$ of (9.1).

Proof. Clearly (ii) implies (i). To prove that (i) implies (ii) it suffices to show that every (almost) exact solution $u$ can be continued up to a noncontinuable one. To this aim, we will make use of the Brezis-Browder Theorem 2.1. Let $\mathcal{S}$ be the set of all (almost) exact solutions to (9.1), defined at least on $[\tau, T)$, and coinciding with $u$ on that interval. On the set $\mathcal{S}$ which, by virtue of (i), is nonempty, we define the binary relation $\preceq$ by $u \preceq v$ if the domain $\left[\tau, T_{v}\right)$ of $v$ is larger than the domain $\left[\tau, T_{u}\right)$ of $u$, i.e., $T_{u} \leq T_{v}$, and $u(t)=v(t)$ for all $t \in\left[\tau, T_{u}\right)$. Clearly $\preceq$ is a preorder on $\mathcal{S}$. Next, let $\left(u_{m}\right)_{m}$ be an increasing sequence in $\mathcal{S}$, and let us denote by $\left[\tau, T_{m}\right)$ the domain of definition of $u_{m}$. Let $T^{*}=\lim _{m} T_{m}$, which is finite, or not, and let us define $u^{*}:\left[\tau, T^{*}\right) \rightarrow X$ by $u^{*}(t)=u_{m}(t)$ for each $t \in\left[\tau, T_{m}\right)$. Since $\left(T_{m}\right)_{m}$ is increasing and $u_{m}(t)=u_{k}(t)$ for each $m \leq k$ and each $t \in\left[\tau, T_{m}\right)$, $u^{*}$ is well defined and belongs to $\mathcal{S}$. Moreover, $u^{*}$ is an upper bound of $\left(u_{m}\right)_{m}$. Thus each increasing sequence in $\mathcal{S}$ is bounded from above. Moreover, the function $\mathcal{N}: \mathcal{S} \rightarrow \mathbb{R}$, defined by $\mathcal{N}(v)=T_{v}$, for each $v \in \mathcal{S}$, is increasing, and therefore we are in the hypotheses of Theorem 2.1. Accordingly, for $u \in \mathcal{S}$, there exists at least one element $\bar{u} \in \mathcal{S}$ with $u \preceq \bar{u}$ and, in addition, $\bar{u} \preceq \widetilde{u}$ implies $T_{\widetilde{u}}=T_{\bar{u}}$. But this means that $\bar{u}$ is noncontinuable, and, of course, that it extends $u$. The proof is complete. 
Remark 9.1. Notice that in Theorem 9.1 we do not assume $\mathcal{C}$ to be locally closed or $F$ to be u.s.c.

We conclude this section with a result concerning the existence of global solutions. Recall that, if $x, y \in X$, we denote by $[x, y]_{+}$the right directional derivative of the norm calculated at $x$ in the direction $y$, i.e.,

$$
[x, y]_{+}=\lim _{h \downarrow 0} \frac{\|x+h y\|-\|x\|}{h} .
$$

Definition 9.1. A multi-function $F: \mathcal{C} \leadsto X$ is called positively sublinear if there exist three continuous functions $a: \mathbb{R} \rightarrow \mathbb{R}_{+}, b: \mathbb{R} \rightarrow \mathbb{R}_{+}$, and $c: \mathbb{R} \rightarrow \mathbb{R}_{+}$such that

$$
\|f\| \leq a(t)\|\xi\|+b(t)
$$

for each $(t, \xi, f) \in K_{+}^{c}(F)$, where

$$
K_{+}^{c}(F)=\left\{(t, \xi, f) \in \mathcal{C} \times X ;\|\xi\|>c(t), f \in F(t, \xi),[\xi, f]_{+}>0\right\} .
$$

Remark 9.2. There are three notable specific cases in which $F$ is positively sublinear:

(i) when $F$ is bounded on $\mathcal{C}$;

(ii) when $F$ has sublinear growth with respect to its last argument 4

(iii) when $f$ satisfies the "sign condition" $[\xi, f]_{+} \leq 0$ for each $(t, \xi) \in \mathcal{C}$ and $f \in F(t, \xi)$.

Definition 9.2. The set $\mathcal{C}$ is $X$-closed if for each sequence $\left(\left(t_{n}, \xi_{n}\right)\right)_{n}$ in $\mathcal{C}$ with $\lim _{n}\left(t_{n}, \xi_{n}\right)=(t, \xi)$, with $t<T_{\mathcal{C}}$, where $T_{\mathcal{C}}$ is given by (9.2), it follows that $(t, \xi) \in \mathcal{C}$.

A typical example of an $X$-closed set is $\mathrm{C}=I \times K$ with $I$ a nonempty and open to the right interval and $K \subseteq X$ nonempty and closed.

Theorem 9.2. Let $X$ be a Banach space, let $\mathcal{C} \subseteq \mathbb{R} \times X$ be nonempty and let $F: \mathcal{C} \leadsto X$ be a given multi-function. If $\mathcal{C}$ is $X$-closed, $F$ maps bounded subsets in $\mathcal{C}$ into bounded subsets in $X$, is positively sublinear, and $\mathcal{C}$ is (almost) exact viable with respect to $F$, then each (almost) exact solution of (9.1) can be continued up to a global one, i.e., defined on $\left[\tau, T_{\mathcal{C}}\right)$, where $T_{\mathcal{C}}$ is given by (9.2).

Proof. Since $\mathcal{C}$ is (almost) exact viable with respect to $f$, by Theorem 9.1, for each $(\tau, \xi) \in \mathcal{C}$, there exists at least one noncontinuable solution $u:[\tau, T) \rightarrow X$ to (9.1). We will show that $T=T_{\mathrm{e}}$. To this aim, let us assume the contrary, i.e., that $T<T_{\mathcal{C}}$. In particular this means that $T<+\infty$. Integrating from $\tau$ to $t$ the equality $\left[u(s), u^{\prime}(s)\right]_{+}=\frac{d^{+}}{d s}(\|u(\cdot)\|)(s)$ for $s \in[\tau, T)$, we get

$$
\|u(t)\|=\|\xi\|+\int_{\tau}^{t}[u(s), f(s, u(s))]_{+} d s
$$

for each $t \in[\tau, T)$. For each $t \in[\tau, T)$, let us denote by

$$
\begin{aligned}
& E_{t}=\left\{s \in[\tau, t] ;[u(s), f(s, u(s))]_{+}>0 \text { and }\|u(s)\|>c(s)\right\}, \\
& G_{t}=\left\{s \in[\tau, t] ;[u(s), f(s, u(s))]_{+} \leq 0\right\}, \\
& H_{t}=\{s \in[\tau, t] ;\|u(s)\| \leq c(s)\} .
\end{aligned}
$$

\footnotetext{
${ }^{4}$ This means that there exist two continuous functions $a: \mathbb{R} \rightarrow \mathbb{R}_{+}$and $b: \mathbb{R} \rightarrow \mathbb{R}_{+}$such that $\|f\| \leq a(t)\|\xi\|+b(t)$ for each $(t, \xi) \in \mathcal{C}$ and $f \in F(t, \xi)$.
} 
Since $[u, v]_{+} \leq\|v\|$ for each $u, v \in X$, we get

$$
\begin{aligned}
\|u(t)\| & \leq\|\xi\|+\int_{E_{t}}[a(s)\|u(s)\|+b(s)] d s+\int_{H_{t} \backslash G_{t}}[u(s), f(s, u(s))]_{+} d s \\
& \leq\|\xi\|+\int_{E_{t}}[a(s)\|u(s)\|+b(s)] d s+\int_{H_{t}}\|f(s, u(s))\| d s .
\end{aligned}
$$

But $f$ maps bounded subsets in $\mathcal{C}$ into bounded subsets in $X$, and therefore there exists $m>0$ such that

$$
\sup \left\{\|f(s, u(s))\| ; s \in H_{t}, t \in[\tau, T)\right\} \leq m .
$$

Hence,

$$
\|u(t)\| \leq\|\xi\|+(T-\tau) m+\int_{\tau}^{T} b(s) d s+\int_{\tau}^{T} a(s)\|u(s)\| d s .
$$

By the Gronwall Lemma, it follows that $u$ is bounded on $[\tau, T)$. Accordingly, $f(\cdot, u(\cdot))$ is bounded on $[\tau, T)$ and so there exists $\lim _{t \uparrow T} u(t)=u^{*}$. Since $\mathcal{C}$ is $X$ closed and $T<T_{\mathcal{E}}$, it follows that $\left(T, u^{*}\right) \in \mathcal{E}$. Using this observation and recalling that $\mathcal{C}$ is viable with respect to $f$, we conclude that $u$ can be continued to the right of $T$. But this is absurd, because $u$ is noncontinuable. This contradiction can be eliminated only if $T=T_{\mathcal{C}}$, and this achieves the proof.

\section{SEmilinear EVOlution INCLUSIONS; NECESSARY CONDITIONS FOR MILD VIABILITY}

Let $X$ be a real Banach space, let $A: D(A) \subseteq X \rightarrow X$ be the infinitesimal generator of a $C_{0}$-semigroup, $\{S(t): X \rightarrow X ; t \geq 0\}, K$ a nonempty subset in $X$ and $F: K \leadsto X$ a given multi-function.

Definition 10.1. By a mild solution of the autonomous multi-valued semilinear Cauchy problem

$$
\left\{\begin{array}{l}
u^{\prime}(t) \in A u(t)+F(u(t)) \\
u(0)=\xi
\end{array}\right.
$$

on $[0, T]$, we mean a continuous function $u:[0, T] \rightarrow K$ for which there exists $f \in L^{1}(0, T ; X)$ such that $f(s) \in F(u(s))$ a.e. for $s \in[0, T]$ and

$$
u(t)=S(t) \xi+\int_{0}^{t} S(t-s) f(s) d s
$$

for each $t \in[0, T]$.

Definition 10.2. The set $K \subseteq X$ is mild viable with respect to $A+F$ if for each $\xi \in K$, there exists $T>0$ such that the Cauchy problem (10.1) has at least one mild solution $u:[0, T] \rightarrow K$.

Let $E \subseteq X$ be nonempty. We denote by

$$
\mathcal{E}=\left\{f \in L^{1}\left(\mathbb{R}_{+} ; X\right) ; f(s) \in E \text { a.e. for } s \in \mathbb{R}_{+}\right\} .
$$

Definition 10.3. Let $A: D(A) \subseteq X \rightarrow X$ be the infinitesimal generator of a $C_{0^{-}}$ semigroup, $\{S(t): X \rightarrow X ; t \geq 0\}, K$ a subset in $X$ and $\xi \in K$. The set $E \subseteq X$ is 
$A$-quasi-tangent to the set $K$ at the point $\xi \in K$ if for each $\rho>0$, we have

$$
\liminf _{h \downarrow 0} \frac{1}{h} \operatorname{dist}\left(S_{\mathcal{E}}(h) \xi ; K \cap D(\xi, \rho)\right)=0,
$$

where

$$
S_{\mathcal{E}}(h) \xi=\left\{S(h) \xi+\int_{0}^{h} S(h-s) f(s) d s ; f \in \mathcal{E}\right\} .
$$

We denote by $\mathcal{Q T S}_{K}^{A}(\xi)$ the class of all $A$-quasi-tangent sets to $K$ at $\xi \in K$.

Another $A$-tangency concept is introduced below.

Definition 10.4. We say that a set $E$ is $A$-tangent to $K$ at $\xi$ if, for each $\rho>0$, we have

$$
\liminf _{h \downarrow 0} \frac{1}{h} \operatorname{dist}\left(S_{E}(h) \xi ; K \cap D(\xi, \rho)\right)=0,
$$

where $S_{E}(h) \xi=\left\{S(h) \xi+\int_{0}^{h} S(h-s) \eta d s ; \eta \in E\right\}$. We denote the class of all $A$-tangent sets to $K$ at $\xi \in K$ by $\mathcal{T S}_{K}^{A}(\xi)$.

Since $E$ can be identified with the subset of a.e. constant elements in $\mathcal{E}$, it readily follows that

$$
\mathcal{T S}_{K}^{A}(\xi) \subseteq \mathcal{Q} \mathcal{T} \mathcal{S}_{K}^{A}(\xi)
$$

Remark 10.1. Let $K \subseteq X, \xi \in K$ and $E \subseteq X$. Then $E \in \mathcal{Q T S}_{K}^{A}(\xi)$ if and only if for each $\varepsilon>0, \rho>0$ and $\delta>0$ there exist $h \in(0, \delta), p \in D(0, \varepsilon)$ and $f \in \mathcal{E}$ such that

$$
S(h) \xi+\int_{0}^{h} S(h-s) f(s) d s+h p \in K \cap D(\xi, \rho) .
$$

Equivalently, $E \in \mathcal{Q T S}_{K}^{A}(\xi)$ if and only if there exist three sequences, $\left(h_{n}\right)_{n}$ in $\mathbb{R}_{+}$ with $h_{n} \downarrow 0,\left(p_{n}\right)_{n}$ in $X$ with $\lim _{n} p_{n}=0$, and $\left(f_{n}\right)_{n} \in \mathcal{E}$, with

$$
\lim _{n} \int_{0}^{h_{n}} S\left(h_{n}-s\right) f_{n}(s) d s=0
$$

and such that

$$
S\left(h_{n}\right) \xi+\int_{0}^{h_{n}} S\left(h_{n}-s\right) f_{n}(s) d s+h_{n} p_{n} \in K .
$$

Remark 10.2. Let $K \subseteq X, \xi \in K$ and $E \in \mathcal{B}(X)$. Then $E \in Q_{\mathcal{T S}}^{A}(\xi)$ if and only if for each $\varepsilon>0$ there exist $\delta \in(0, \varepsilon], p \in X$ with $\|p\| \leq \varepsilon$ and $f \in \mathcal{E}$ such that

$$
S(\delta) \xi+\int_{0}^{\delta} S(\delta-s) f(s) d s+\delta p \in K .
$$

Equivalently, $E \in \mathcal{Q T S}_{K}^{A}(\xi)$ if and only if there exist three sequences, $\left(h_{n}\right)_{n}$ in $\mathbb{R}_{+}$, with $h_{n} \downarrow 0,\left(f_{n}\right)_{n}$ in $\mathcal{E}$ and $\left(p_{n}\right)_{n}$ in $X$ with $\lim _{n} p_{n}=0$ and such that

$$
S\left(h_{n}\right) \xi+\int_{0}^{h_{n}} S\left(h_{n}-s\right) f_{n}(s) d s+h_{n} p_{n} \in K
$$

for $n=1,2, \ldots$. 
Proposition 10.1. Let $K \subseteq X, \xi \in K$ and $E \in \mathcal{B}(X)$. We have

(i) if $S(t) K \subseteq K$ for each $t>0$, then $\{0\} \in \mathcal{Q T S}_{K}^{A}(\xi)$;

(ii) if $E \subseteq D$ and $E \in Q \mathcal{T S} \mathcal{S}_{K}^{A}(\xi)$, then $D \in Q \mathcal{T} \mathcal{S}_{K}^{A}(\xi)$;

(iii) if $S(t) K \subseteq K$ for each $t>0$ and $0 \in E$, then $E \in \mathcal{Q T S}_{K}^{A}(\xi)$;

(iv) $E \in \mathcal{Q T S}_{K}^{\bar{A}}(\xi)$ if and only if $\bar{E} \in \mathcal{Q T} \mathcal{S}_{K}^{A}(\xi)$;

(v) Let $\eta \in X$. Then $\eta \in \mathcal{T}_{K}^{A}(\xi)$ if and only if $\{\eta\} \in Q \mathcal{Q T} \mathcal{S}_{K}^{A}(\xi)$;

(vi) if $E$ is compact and convex, then $E \in \mathcal{Q T S}_{K}(\xi)$ if and only if there exists $\eta \in E$ such that $\eta \in \mathcal{T}_{K}^{A}(\xi)$.

Proof. Except for (iv) and (vi), which will be proved below, the remaining properties are direct consequences of Remark 10.2, Let us observe that (iv) follows from the remark that each measurable function $f: \mathbb{R}_{+} \rightarrow \bar{E}$ can be approximated uniformly with countably-valued functions taking values in $E$. See Theorem 2.2 .

To prove (vi), let $E$ be compact and convex and let $\left(f_{n}\right)_{n}$ in $\mathcal{E}, h_{n} \downarrow 0$ and $\left(p_{n}\right)_{n}$ with $\lim _{n} p_{n}=0$ as in Remark 10.2, i.e., with

$$
S\left(h_{n}\right) \xi+\int_{0}^{h_{n}} S\left(h_{n}-s\right) f_{n}(s) d s+h_{n} p_{n} \in K
$$

for $n=1,2, \ldots$ For $n=1,2, \ldots$, let us define

$$
\eta_{n}=\frac{1}{h_{n}} \int_{0}^{h_{n}} f_{n}(s) d s .
$$

Since $E$ is convex and closed, we have that $\eta_{n} \in E$ for $n=1,2, \ldots$ But $E$ is compact and thus, we may assume with no loss of generality that there exists $\eta \in E$ such that

$$
\lim _{n} \eta_{n}=\eta
$$

Since $E$ is compact, we have

$$
\begin{aligned}
& \lim _{n}\left\|\frac{1}{h_{n}} \int_{0}^{h_{n}} S\left(h_{n}-s\right) f_{n}(s) d s-\frac{1}{h_{n}} \int_{0}^{h_{n}} S\left(h_{n}-s\right) \eta d s\right\| \\
\leq & \lim _{n} \frac{1}{h_{n}}\left\|\int_{0}^{h_{n}}\left[S\left(h_{n}-s\right) f_{n}(s)-f_{n}(s)\right] d s\right\|+\lim _{n}\left\|\eta_{n}-\eta\right\|=0 .
\end{aligned}
$$

The conclusion follows from Remark 10.1 and (v). This completes the proof.

Remark 10.3. From (vi) in Proposition 10.1 it follows that if $E$ is convex and compact, then $E \in Q \mathcal{T} \mathcal{S}_{K}^{A}(\xi)$ if and only if $E \cap \mathcal{T}_{K}^{A}(\xi) \neq \emptyset$.

Remark 10.4. The result obtained by replacing $2 \mathcal{T S} \mathcal{S}_{K}^{A}(\xi)$ in Proposition 10.1 by $\mathcal{T S}_{K}^{A}(\xi)$ holds true with the special mention that in this case, in (vi), there is no need to assume that $E$ is convex.

Definition 10.5. A set $C \subseteq X$ is quasi-weakly (relatively) compact if, for each $r>0, C \cap D(0, r)$ is weakly (relatively) compact.

Remark 10.5. Each quasi-weakly compact set is closed. If $X$ is reflexive, then each closed and convex set $C \subseteq X$ is quasi-weakly compact. See Theorems 2.4 and 2.5 .

Theorem 10.1. Let $X$ be a Banach space, $A: D(A) \subseteq X \rightarrow X$ the infinitesimal generator of a $C_{0}$-semigroup, $\{S(t): X \rightarrow X ; t \geq 0\}, K$ a nonempty subset in $X$ and $F: K \leadsto X$ a nonempty-valued multi-function. If $K$ is mild viable with 
respect to $A+F$, then, for each $\xi \in K$ at which $F$ is u.s.c. and $F(\xi)$ is convex and quasi-weakly compact, we have

$$
F(\xi) \in \mathcal{Q T S}_{K}^{A}(\xi)
$$

To prove Theorem 10.1 we need

Lemma 10.1. Let $X$ be a Banach space, let $C$ be a convex and quasi-weakly compact subset in $X$, let $\rho>0$ and let $v \in L^{1}(0, T ; X)$, with $v(t) \in C+D(0, \rho)$ a.e. for $t \in[0, T]$. Then there exist two measurable functions $f:[0, T] \rightarrow C$ and $g:[0, T] \rightarrow D(0, \rho)$ such that $f \in L^{1}(0, T ; X)$ and $v(s)=f(s)+g(s)$ a.e. for $s \in[0, T]$.

Proof. Since $v \in L^{1}(0, T ; X)$, by Theorem 2.2. there exists $\rho_{n} \downarrow \rho$ and a sequence of countably-valued functions $\left(v_{n}\right)_{n}, v_{n}:[0, T] \rightarrow C+D\left(0, \rho_{n}\right)$ such that $\lim _{n} v_{n}=v$ a.e. uniformly on $[0, T]$ and in $L^{1}(0, T ; X)$, too. See Definition 2.2. Moreover, there exist two sequences of countably-valued functions $\left(f_{n}\right)_{n}$ and $\left(g_{n}\right)_{n}, f_{n}:[0, T] \rightarrow C$, $g_{n}:[0, T] \rightarrow D\left(0, \rho_{n}\right)$ such that $v_{n}=f_{n}+g_{n}$, for $n=1,2, \ldots$ As $\lim _{n} v_{n}=v$ a.e. uniformly on $[0, T]$, we conclude that

$$
\begin{gathered}
\left\|f_{n}(t)\right\| \leq\left\|v_{n}(t)\right\|+\left\|g_{n}(t)\right\| \leq\|v(t)\|+\left\|v_{n}(t)-v(t)\right\|+\rho_{n} \\
\leq\|v(t)\|+M,
\end{gathered}
$$

a.e. for $t \in[0, T]$, where $M>0$ is independent of $n=1,2, \ldots$ From (10.8), as $C$ is quasi-weakly compact and $v \in L^{1}(0, T ; X)$, we deduce that, for each $\varepsilon>0$, there exist a weakly compact subset $C_{\varepsilon}$ of $C$ and a set $E_{\varepsilon} \subseteq[0, T]$ such that, for $n=1,2, \ldots, f_{n}\left(E_{\varepsilon}\right) \subseteq C_{\varepsilon} 5$ and $\mu\left([0, T] \backslash E_{\varepsilon}\right) \leq \varepsilon$. Indeed, let $\varepsilon>0$ be arbitrary, let $r_{\varepsilon}>0$ be such that

$$
\frac{\|v\|_{L^{1}(0, T ; X)}+T M}{r_{\varepsilon}} \leq \varepsilon
$$

and let $C_{\varepsilon}=C \cap D\left(0, r_{\varepsilon}\right)$. Finally, let

$$
E_{\varepsilon}=\left\{t \in[0, T] ;\|v(t)\|+M \leq r_{\varepsilon}\right\} .
$$

Obviously, $C_{\varepsilon}$ is weakly compact and, in view of (10.8), $f_{n}\left(E_{\varepsilon}\right) \subseteq C_{\varepsilon}$ for $n=$ $1,2, \ldots$ To prove that $\mu\left([0, T] \backslash E_{\varepsilon}\right) \leq \varepsilon$, let us observe that

$$
\begin{aligned}
& r_{\varepsilon} \mu\left([0, T] \backslash E_{\varepsilon}\right) \leq \int_{[0, T] \backslash E_{\varepsilon}}(\|v(s)\|+M) d s \\
& \leq \int_{0}^{T}(\|v(s)\|+M) d s=\|v\|_{L^{1}(0, T ; X)}+T M .
\end{aligned}
$$

From this inequality and (10.9), we deduce $\mu\left([0, T] \backslash E_{\varepsilon}\right) \leq \varepsilon$, as claimed. So, the family $\left\{f_{n} ; n=1,2, \ldots\right\}$ satisfies the hypotheses of Theorem 2.6. Therefore, it is weakly relatively compact in $L^{1}([0, T] ; X)$. In view of Theorem $[2.4$, it is weakly sequentially compact. Hence, we may assume with no loss of generality that $\lim _{n} f_{n}=f$ weakly in $L^{1}(0, T ; X)$. Then, in view of Proposition 2.1, there exists $\left(\widetilde{f}_{n}\right)_{n}$ with $\widetilde{f}_{n} \in \operatorname{conv}\left\{f_{k} ; k \geq n\right\}$, such that $\lim _{n} \widetilde{f}_{n}=f$ in $L^{1}(0, T ; X)$. So, on a subsequence at least, $\lim _{n} \widetilde{f}_{n}(s)=f(s)$ a.e. for $s \in[0, T]$. But $C$ is convex and closed. See Remark 10.5. Thus $f(s) \in C$ a.e. for $s \in[0, T]$. Since

\footnotetext{
${ }^{5}$ In fact we have $f_{n}(t) \in C_{\varepsilon}$ a.e. for $t \in E_{\varepsilon}$, but redefining the functions $f_{n}$ on a set of null measure we arrive at $f_{n}\left(E_{\varepsilon}\right) \subseteq C_{\varepsilon}$ without affecting the rest of the properties of $f_{n}$ needed in what follows.
} 
$v_{n}=f_{n}+g_{n}$ and $\left(f_{n}\right)_{n}$ converges weakly in $L^{1}(0, T ; X)$, while $\left(v_{n}\right)_{n}$ converges strongly in $L^{1}(0, T ; X)$ to $v$, we conclude that $\left(g_{n}\right)_{n}$ converges weakly in $L^{1}(0, T ; X)$ to $g=v-f$. Redefining both $f$ and $g$ on a set of null Lebesgue measure, if necessary, we may assume that $f(s) \in C, g(s) \in D(0, \rho)$ for each $s \in[0, T]$ and $v(s)=f(s)+g(s)$ a.e. for $s \in[0, T]$. As $f$ and $g$ are measurable, the proof is complete.

We can now proceed to the proof of Theorem 10.1 .

Proof. Let $\xi \in K$. Since $K$ is mild viable with respect to $A+F$ there exists at least one mild solution $u:[0, T] \rightarrow K$ of (10.1). Let $f \in L^{1}(0, T ; X)$ be the function given by Definition 10.1. As $u$ is continuous at $t=0$ and $F$ is u.s.c. at $u(0)=\xi$, it follows that for each $\rho>0$ there exists $\delta(\rho)>0$ such that

$$
f(s) \in F(\xi)+D(0, \rho)
$$

a.e. for $s \in[0, \delta(\rho)]$. Then, if $\left(\rho_{n}\right)_{n}$ is a sequence in $(0,1), \rho_{n} \downarrow 0$, there exists $h_{n} \downarrow 0$ such that

$$
f(s) \in F(\xi)+D\left(0, \rho_{n}\right)
$$

for $n=1,2, \ldots$ and a.e. for $s \in\left[0, h_{n}\right]$. So, for $n=1,2, \ldots$, there exist $f_{n}$ and $g_{n}$ with $f_{n}(s) \in F(\xi), g_{n}(s) \in D\left(0, \rho_{n}\right)$ and

$$
f(s)=f_{n}(s)+g_{n}(s)
$$

a.e. for $s \in\left[0, h_{n}\right]$. Since $F(\xi)$ is convex and quasi-weakly compact, thanks to Lemma 10.1, we may assume without loss of generality that both $f_{n}$ and $g_{n}$ are integrable. Let us observe that

$$
\begin{gathered}
\left\|u\left(h_{n}\right)-S\left(h_{n}\right) \xi-\int_{0}^{h_{n}} S\left(h_{n}-s\right) f_{n}(s) d s\right\| \\
=\left\|S\left(h_{n}\right) \xi+\int_{0}^{h_{n}} S\left(h_{n}-s\right) f(s) d s-S\left(h_{n}\right) \xi-\int_{0}^{h_{n}} S\left(h_{n}-s\right) f_{n}(s) d s\right\| \\
\leq M h_{n} e^{a h_{n}} \rho_{n},
\end{gathered}
$$

for $n=1,2, \ldots$, where $M \geq 1$ and $a \geq 0$ are such that

$$
\|S(t) x\| \leq M e^{a t}\|x\|
$$

for every $x \in X$ and $t \geq 0$. See Vrabie 24, Theorem 2.3.1, p. 41. In fact $a \in \mathbb{R}$, but, for our purposes, we may always assume that $a \geq 0$. Now, let us denote by

$$
p_{n}=\frac{1}{h_{n}}\left(u\left(h_{n}\right)-S\left(h_{n}\right) \xi-\int_{0}^{h_{n}} S\left(h_{n}-s\right) f_{n}(s) d s\right) .
$$

Since $\lim _{n} p_{n}=0$ and

$$
\lim _{n} \int_{0}^{h_{n}} S\left(h_{n}-s\right) f_{n}(s) d s=\lim _{n}\left(u\left(h_{n}\right)-S\left(h_{n}\right) \xi-h_{n} p_{n}\right)=0
$$

and

$$
S\left(h_{n}\right) \xi+\int_{0}^{h_{n}} S\left(h_{n}-s\right) f_{n}(s) d s+h_{n} p_{n}=u\left(h_{n}\right) \in K
$$

for $n=1,2, \ldots$, from Remark 10.1 , it follows that $F(\xi) \in 2 \mathcal{T S}_{K}^{A}(\xi)$ and this achieves the proof. 
Remark 10.6. In Theorem 10.1, we can replace the assumption "quasi-weakly compact" by "quasi-weakly relatively compact" to obtain the very same conclusion.

If $X$ is reflexive, we have

Theorem 10.2. Let $X$ be reflexive, let $A: D(A) \subseteq X \rightarrow X$ be the infinitesimal generator of a $C_{0}$-semigroup $\{S(t): X \rightarrow X ; t \geq 0\}, K$ a nonempty subset in $X$ and $F: K \leadsto X$ a nonempty-valued multi-function. If $K$ is mild viable with respect to $A+F$, then, for each $\xi \in K$ at which $F$ is u.s.c. and $F(\xi)$ is convex and closed, we have (10.6).

Coming back to general Banach spaces, if $F$ is compact-valued, then, instead of tangent sets, we can use tangent vectors to get a necessary condition in order for $K$ to be mild viable with respect to $A+F$. Namely, we have

Theorem 10.3. Let $X$ be a Banach space, $A: D(A) \subseteq X \rightarrow X$ the infinitesimal generator of a $C_{0}$-semigroup $\{S(t): X \rightarrow X ; t \geq 0\}, K$ a nonempty subset in $X$ and $F: K \leadsto X$ a nonempty-valued multi-function. If $K$ is mild viable with respect to $A+F$, then at each point $\xi \in K$, at which $F$ is u.s.c. and $F(\xi)$ is convex and compact, we have

$$
F(\xi) \cap \mathcal{T}_{K}^{A}(\xi) \neq \emptyset .
$$

Proof. The conclusion follows from Theorem 10.1 and Remark 10.3 .

\section{SUFFICIENT CONDITIONS FOR MILD VIABILITY}

In order to handle several apparently different cases in a unitary frame, we introduce:

Definition 11.1. Let $A: D(A) \subseteq X \rightarrow X$ be the infinitesimal generator of a $C_{0}$-semigroup $\{S(t): X \rightarrow X ; t \geq 0\}$ and $F: K \leadsto X$ a multi-function. We say that $A+F$ is locally of compact type if $F$ is u.s.c. and, for each $\xi \in K$, there exist $\rho>0$, a continuous function $\ell: \mathbb{R}_{+} \rightarrow \mathbb{R}_{+}$and a uniqueness function $\omega: \mathbb{R}_{+} \rightarrow \mathbb{R}_{+}$, such that, for each $C \subseteq D(\xi, \rho) \cap K, F(C)$ is bounded and

$$
\beta(S(t) F(C)) \leq \ell(t) \omega(\beta(C))
$$

for each $t>0$.

Remark 11.1. As in the single-valued case, one may easily verify that $A+F$ is locally of compact type whenever:

(i) $F$ is locally $\beta$-compact (see Definition 5.2 );

(ii) $F$ is u.s.c., has nonempty and closed values, is locally bounded 6 and the $C_{0}$-semigroup generated by $A$ is compact.

The main sufficient conditions for mild viability are:

Theorem 11.1. Let $X$ be a Banach space, $A: D(A) \subseteq X \rightarrow X$ the infinitesimal generator of a $C_{0}$-semigroup $\{S(t): X \rightarrow X ; t \geq 0\}, K$ a nonempty and locally closed subset in $X$ and $F: K \leadsto X$ a nonempty, weakly compact, closed and convex

\footnotetext{
${ }^{6}$ This happens for instance whenever $X$ is reflexive, $F$ is u.s.c. and has nonempty, closed, convex and bounded values. Indeed, $F$ is strongly-weakly u.s.c., being strongly-strongly u.s.c. Furthermore, since $X$ is reflexive, each bounded, closed convex set is weakly compact, and thus, by Lemma $2.1 F$ is locally bounded.
} 
valued multi-function such that $A+F$ is locally of compact type. If, for each $\xi \in K$, we have

$$
F(\xi) \in \mathcal{Q T S}_{K}^{A}(\xi),
$$

then $K$ is mild viable with respect to $A+F$.

Theorem 11.2. Let $X$ be a Banach space, $A: D(A) \subseteq X \rightarrow X$ the infinitesimal generator of a $C_{0}$-semigroup $\{S(t): X \rightarrow X ; t \geq 0\}, K$ a nonempty and locally closed subset in $X$ and $F: K \leadsto X$ a nonempty, closed and convex valued, locally $\beta$-compact multi-function. Then $K$ is mild viable with respect to $A+F$ if and only if, for each $\xi \in K$, the tangency condition $F(\xi) \cap \mathcal{T}_{K}^{A}(\xi) \neq \emptyset$ is satisfied.

Theorem 11.3. Let $X$ be a Banach space, $A: D(A) \subseteq X \rightarrow X$ the infinitesimal generator of a compact $C_{0}$-semigroup $\{S(t): X \rightarrow X ; t \geq 0\}, K$ a nonempty and locally closed subset in $X$ and $F: K \leadsto X$ a strongly-weakly u.s.c. multi-function with nonempty, weakly compact and convex values. If, for each $\xi \in K$, the tangency condition (11.1) is satisfied, then $K$ is mild viable with respect to $A+F$.

Theorem 11.4. Let $X$ be a Banach space, $A: D(A) \subseteq X \rightarrow X$ the infinitesimal generator of a $C_{0}$-semigroup $\{S(t): X \rightarrow X ; t \geq 0\}, K$ a nonempty and locally compact subset in $X$ and $F: K \leadsto X$ a strongly-weakly u.s.c. multi-function with nonempty, weakly compact and convex values. If, for each $\xi \in K$, the tangency condition (11.1) is satisfied, then $K$ is mild viable with respect to $A+F$.

\section{EXISTENCE OF $\varepsilon$-APPROXIMATE MiLD SOLUTiOnS}

The proof of the sufficiency of Theorems 11.1 and 11.4 is based on the following existence result concerning $\varepsilon$-approximate solutions for the Cauchy problem (10.1). We notice that Lemma 12.1 below, which is a nontrivial extension of Lemma 6.1 to the semilinear case, is inspired from Cârjă-Vrabie [8].

Lemma 12.1. Let $X$ be a Banach space, $A: D(A) \subseteq X \rightarrow X$ the infinitesimal generator of a $C_{0}$-semigroup $\{S(t): X \rightarrow X ; t \geq 0\}, K$ a nonempty and locally closed subset in $X$ and $F: K \leadsto X$ a given nonempty-valued and locally bounded multi-function satisfying the tangency condition (11.1). Let $\xi \in K$ be arbitrary and let $r>0$ be such that $D(\xi, r) \cap K$ is closed. Then, there exist $\rho \in(0, r]$ and $T>0$ such that, for each $\varepsilon \in(0,1)$, there exist $\sigma:[0, T] \rightarrow[0, T]$ nondecreasing, $\theta:\{(t, s) ; 0 \leq s<t \leq T\} \rightarrow[0, T]$ measurable, $g:[0, T] \rightarrow X$ and $f:[0, T] \rightarrow X$ Bochner integrable and $u:[0, T] \rightarrow X$ continuous such that:

(i) $s-\varepsilon \leq \sigma(s) \leq s$ for each $s \in[0, T]$;

(ii) $u(\sigma(s)) \in D(\xi, \rho) \cap K$ for each $s \in[0, T]$ and $u(T) \in D(\xi, \rho) \cap K$;

(iii) $\|g(s)\| \leq \varepsilon$ for each $s \in[0, T]$ and $f(s) \in F(u(\sigma(s)))$ a.e. for $s \in[0, T]$;

(iv) $\theta(t, s) \leq t$ for each $0 \leq s<t \leq T$ and $t \mapsto \theta(t, s)$ is nonexpansive on $(s, T]$;

(v) $u(t)=S(t) \xi+\int_{0}^{t} S(t-s) f(s) d s+\int_{0}^{t} S(\theta(t, s)) g(s) d s$ for each $t \in[0, T]$;

(vi) $\|u(t)-u(\sigma(t))\| \leq \varepsilon$ for each $t \in[0, T]$.

Definition 12.1. An $\varepsilon$-approximate mild solution of (10.1) on $[0, T]$ is a 5-tuple $(\sigma, \theta, g, f, u)$ satisfying (i) (vi) in Lemma 12.1 .

Proof. Let $\xi \in K$ be arbitrary and let $r>0$ be such that $D(\xi, r) \cap K$ is closed. Let us choose $\rho \in(0, r], N>0, M \geq 1$ and $a \geq 0$ such that

$$
\|y\| \leq N
$$


for every $x \in D(\xi, \rho) \cap K$ and $y \in F(x)$, and (10.10). The existence of $\rho>0$ and $N>0$ satisfying (12.1) is ensured by the fact that $F$ is locally bounded. Since $t \mapsto S(t) \xi$ is continuous at $t=0$ and $S(0) \xi=\xi$, we may find a sufficiently small $T>0$ such that

$$
\sup _{t \in[0, T]}\|S(t) \xi-\xi\|+T M e^{a T}(N+1) \leq \rho .
$$

Let $\varepsilon \in(0,1)$ be arbitrary but fixed. We begin by showing the existence of an $\varepsilon$ approximate mild solution on an interval $[0, \delta]$ with $\delta \in(0, T]$. As, for every $\xi \in K$, $F$ satisfies the tangency condition (11.1), it follows that there exist $\delta \in(0, T]$, $f \in \mathcal{F}(\xi)]^{7}$ and $p \in X$ with $\|p\| \leq \varepsilon$, such that

$$
S(\delta) \xi+\int_{0}^{\delta} S(\delta-s) f(s) d s+\delta p \in K .
$$

We continue by showing how to define the functions $\sigma, \theta, g, f$ and $u$. First, $f$ is defined as above. Next, let $\sigma:[0, \delta] \rightarrow[0, \delta], g:[0, \delta] \rightarrow X$ and $\theta:\{(t, s) ; 0 \leq$ $s<t \leq \delta\} \rightarrow[0, \delta]$ be given by

$$
\begin{aligned}
& \sigma(s)=0, \\
& g(s)=p, \\
& \theta(t, s)=0
\end{aligned}
$$

and let $u:[0, \delta] \rightarrow X$ be defined by

$$
u(t)=S(t) \xi+\int_{0}^{t} S(t-s) f(s) d s+t p
$$

for each $t \in[0, \delta]$.

We will show that $(\sigma, \theta, g, f, u)$ is an $\varepsilon$-approximate mild solution to the Cauchy problem (10.1) on the interval $[0, \delta]$. Clearly $\sigma$ is nondecreasing, $g$ and $f$ are Bochner integrable, $\theta$ is measurable and $t \mapsto \theta(t, s)$ is nonexpansive on $[0, \delta]$ and $u$ is continuous. The conditions (i), (iii), (iv) and (v) are obviously fulfilled. To prove (ii), let us first observe that, as $u(\sigma(s))=u(0)=\xi$ for each $s \in[0, \delta]$, we have $u(\sigma(s)) \in D(\xi, \rho) \cap K$. Moreover, since $\varepsilon<1$, from (12.1) and (10.10), we get

$$
\begin{gathered}
\|u(t)-\xi\| \leq\|S(t) \xi-\xi\|+\int_{0}^{t}\|S(t-s)\|_{\mathcal{L}(X)}\|f(s)\| d s \\
+\int_{0}^{t}\|S(\theta(t, s))\|_{\mathcal{L}(X)}\|p\| d s \leq \sup _{t \in[0, \delta]}\|S(t) \xi-\xi\|+\delta M e^{a \delta}(N+1)
\end{gathered}
$$

for each $t \in[0, \delta]$. Next, since $\delta<T$, by (12.2), we have

$$
\|u(\delta)-\xi\| \leq \sup _{t \in[0, \delta]}\|S(t) \xi-\xi\|+\delta M e^{a \delta}(N+1) \leq \rho .
$$

Combining (12.3) with the last inequality, we get $u(\delta) \in D(\xi, \rho) \cap K$ and thus (ii) is satisfied. Diminishing $\delta>0$, if necessary, by (12.4), we may assume that

$$
\|u(t)-u(\sigma(t))\| \leq \varepsilon
$$

for each $t \in[0, \delta]$ and thus (vi) is also satisfied. We emphasize that the reason we can do this is because (12.4) is "independent" of $p$ which, of course, may change with $\delta$. Therefore $(\sigma, \theta, g, f, u)$ is an $\varepsilon$-approximate mild solution of (10.1) on $[0, \delta]$.

\footnotetext{
${ }^{7}$ We recall that $\mathcal{F}(\xi)=\left\{f \in L^{1}\left(\mathbb{R}_{+} ; X\right) ; f(s) \in F(\xi)\right.$ a.e. for $\left.s \in \mathbb{R}_{+}\right\}$.
} 
In the second step we will prove the existence of an $\varepsilon$-approximate mild solution for (10.1) defined on the whole interval $[0, T]$. To this aim we shall make use of the Brezis-Browder Theorem 2.1. We denote by $D(c)$ the set

$$
D(c)=[0, c] \times\{(t, s) ; 0 \leq s<t \leq c\} \times[0, c] \times[0, c] \times[0, c],
$$

with $c>0$, and by $\mathcal{S}$ the set of all $\varepsilon$-approximate mild solutions to the problem (10.1), defined on $D(c)$, with $c \leq T$.

On the set $\mathcal{S}$ we introduce a preorder relation "․" as follows: we say that $\left(\sigma_{1}, \theta_{1}, g_{1}, f_{1}, u_{1}\right)$, defined on $D\left(c_{1}\right)$, and $\left(\sigma_{2}, \theta_{2}, g_{2}, f_{2}, u_{2}\right)$, defined on $D\left(c_{2}\right)$, satisfy

$$
\left(\sigma_{1}, \theta_{1}, g_{1}, f_{1}, u_{1}\right) \preceq\left(\sigma_{2}, \theta_{2}, g_{2}, f_{2}, u_{2}\right)
$$

if $c_{1} \leq c_{2}, \sigma_{1}(t)=\sigma_{2}(t), g_{1}(t)=g_{2}(t)$ and $f_{1}(t)=f_{2}(t)$ for $t \in\left[0, c_{1}\right]$ and $\theta_{1}(t, s)=\theta_{2}(t, s)$ for each $0 \leq s<t \leq c_{1}$.

Let $\mathcal{L}$ be an increasing sequence in $\mathcal{S}$,

$$
\mathcal{L}=\left(\left(\sigma_{i}, \theta_{i}, g_{i}, f_{i}, u_{i}\right): D\left(c_{i}\right) \rightarrow\left[0, c_{i}\right] \times\left[0, c_{i}\right] \times X \times X \times X\right)_{i} .
$$

We define an upper bound of $\mathcal{L}$ as follows. First, set

$$
c^{*}=\sup \left\{c_{i} ; i=1,2, \ldots\right\} .
$$

If $c^{*}=c_{i}$ for some $i=1,2, \ldots$, then $\left(\sigma_{i}, \theta_{i}, g_{i}, f_{i}, u_{i}\right)$ is an upper bound for $\mathcal{L}$. If $c_{i}<c^{*}$ for $i=1,2, \ldots$, we show that there exists $\left(\sigma^{*}, \theta^{*}, g^{*}, f^{*}, u^{*}\right)$ in $\mathcal{S}$, defined on $\left[0, c^{*}\right]$, and satisfying

$$
\left(\sigma_{m}, \theta_{m}, g_{m}, f_{m}, u_{m}\right) \preceq\left(\sigma^{*}, \theta^{*}, g^{*}, f^{*}, u^{*}\right),
$$

for $m=1,2, \ldots$.

First, we know that all the functions in the set $\left\{\sigma_{m} ; m=1,2, \ldots\right\}$ are nondecreasing, with values in $\left[0, c^{*}\right]$, and satisfy $\sigma_{m}\left(c_{m}\right) \leq \sigma_{p}\left(c_{p}\right)$ for $m, p=1,2, \ldots$ with $m \leq p$. So, there exists

$$
\lim _{m} \sigma_{m}\left(c_{m}\right) \in\left[0, c^{*}\right] .
$$

Now let $m, k \in\{1,2, \ldots\}$ be arbitrary with $m \geq k$. For each $s \in\left[0, c_{k}\right)$, we have

$$
\begin{gathered}
\left|\theta_{m}\left(c_{m}, s\right)-\theta_{k}\left(c_{k}, s\right)\right| \\
\leq\left|\theta_{m}\left(c_{m}, s\right)-\theta_{m}\left(c_{k}, s\right)\right|+\left|\theta_{m}\left(c_{k}, s\right)-\theta_{k}\left(c_{k}, s\right)\right| \leq\left|c_{m}-c_{k}\right|,
\end{gathered}
$$

because $t \mapsto \theta_{m}(t, s)$ is nonexpansive on $\left(s, c^{*}\right)$. Accordingly, there exists

$$
\lim _{m} \theta_{m}\left(c_{m}, s\right) \text {. }
$$

We are now ready to define the functions $\sigma^{*}:\left[0, c^{*}\right] \rightarrow\left[0, c^{*}\right]$ by

$$
\begin{gathered}
\sigma^{*}(t)=\left\{\begin{array}{cl}
\sigma_{m}(t) & \text { if, for some } m=1,2, \ldots, t \in\left[0, c_{m}\right] \\
\lim _{m} \sigma_{m}\left(c_{m}\right) & \text { if } t=c^{*},
\end{array}\right. \\
\theta^{*}:\left\{(t, s) ; 0 \leq s<t \leq c^{*}\right\} \rightarrow\left[0, c^{*}\right] \text { by } \\
\theta^{*}(t, s)= \begin{cases}\theta_{m}(t, s) & \text { if, for some } m=1,2, \ldots, 0 \leq s<t \leq c_{m} \\
\lim _{m} \theta_{m}\left(c_{m}, s\right) & \text { if } 0 \leq s<t=c^{*},\end{cases}
\end{gathered}
$$

and $g^{*}:\left[0, c^{*}\right] \rightarrow X$ by

$$
g^{*}(t)=\left\{\begin{array}{cl}
g_{m}(t) & \text { if, for some } m=1,2, \ldots, t \in\left[0, c_{m}\right] \\
0 & \text { if } t=c^{*}
\end{array}\right.
$$


Obviously $\sigma^{*}$ is nondecreasing, $g^{*}$ is Bochner integrable, $\theta^{*}$ is measurable and $t \mapsto \theta^{*}(t, s)$ is nonexpansive on $[s, \delta]$. In order to define $u^{*}$ and $f^{*}$, we will prove first that there exists $\lim _{m} u_{m}\left(c_{m}\right)$. To this aim, let us denote by $\chi_{\left[0, c_{m}\right]}:[0, T] \rightarrow \mathbb{R}_{+}$ the indicator function of $\left[0, c_{m}\right]$, i.e.,

$$
\chi_{\left[0, c_{m}\right]}(s)= \begin{cases}1 & \text { if } s \in\left[0, c_{m}\right] \\ 0 & \text { if } s \in\left(c_{m}, T\right] .\end{cases}
$$

By (v), we have

$$
\begin{aligned}
u_{m}\left(c_{m}\right) & =S\left(c_{m}\right) \xi+\int_{0}^{c^{*}} \chi_{\left[0, c_{m}\right]}(s) S\left(c_{m}-s\right) f_{m}(s) d s \\
& +\int_{0}^{c^{*}} \chi_{\left[0, c_{m}\right]}(s) S\left(\theta_{m}\left(c_{m}, s\right)\right) g_{m}(s) d s .
\end{aligned}
$$

Recalling (i), (ii), (iv) and (vi) and the fact that both $g_{m}$ and $f_{m}$ are Bochner integrable and, by (12.1) and (iii), both are bounded (the former by $N$ and the latter by $\varepsilon$ ), thanks to the Lebesgue Dominated Convergence, we deduce that there exists $\lim _{m} u_{m}\left(c_{m}\right)=\xi^{*}$.

Let us define $u^{*}:\left[0, c^{*}\right] \rightarrow X$ by

$$
u^{*}(t)=\left\{\begin{array}{cl}
u_{m}(t) & \text { if, for some } m=1,2, \ldots, t \in\left[0, c_{m}\right] \\
\xi^{*} & \text { if } t=c^{*}
\end{array}\right.
$$

Furthermore, let us define $f^{*}:\left[0, c^{*}\right] \rightarrow X$ by

$$
f^{*}(t)=\left\{\begin{array}{cl}
f_{m}(t) & \text { if, for some } m=1,2, \ldots, t \in\left[0, c_{m}\right] \\
\eta & \text { if } t=c^{*}
\end{array}\right.
$$

where $\eta \in X$ is arbitrary but fixed.

Clearly $u^{*}$ is continuous on $\left[0, c^{*}\right]$. Moreover, $\left(\sigma^{*}, \theta^{*}, g^{*}, f^{*}, u^{*}\right)$ satisfies (i), (iii), (iv) and (v). Since $u_{m}$ is an $\varepsilon$-approximate mild solution on $\left[0, c_{m}\right]$, by (ii) applied to $u_{m}$, we have $u_{m}\left(c_{m}\right) \in D(\xi, \rho) \cap K$ and since the latter is closed, we have $u^{*}\left(c^{*}\right) \in D(\xi, \rho) \cap K$. Similarly, by (vi) applied to $u_{m}$, we have

$$
\left\|u_{m}(t)-u_{m}\left(\sigma_{m}(t)\right)\right\| \leq \varepsilon
$$

for each $m \in \mathbb{N}$ and $t \in\left[0, c_{m}\right]$ and since $u^{*}$ is continuous, we deduce

$$
\left\|u^{*}(t)-u^{*}\left(\sigma^{*}(t)\right)\right\| \leq \varepsilon
$$

for each $t \in\left[0, c^{*}\right]$. Hence $u^{*}$ and $\sigma^{*}$ satisfy (ii) and (vi). Thus the quintuple $\left(\sigma^{*}, \theta^{*}, g^{*}, f^{*}, u^{*}\right)$ is an $\varepsilon$-approximate mild solution of (10.1) on [0, $\left.c^{*}\right]$ and

$$
\left(\sigma_{m}, \theta_{m}, g_{m}, f_{m}, u_{m}\right) \preceq\left(\sigma^{*}, \theta^{*}, g^{*}, f^{*}, u^{*}\right),
$$

for $m=1,2, \ldots$ Let us define $\mathcal{N}: \mathcal{S} \rightarrow \mathbb{R} \cup\{+\infty\}$ by $\mathcal{N}((\sigma, \theta, g, f, u))=c$, where $D(c)$ is the domain of definition of $(\sigma, \theta, g, f, u)$. Clearly $\mathcal{N}$ satisfies the hypotheses of the Brezis-Browder Theorem 2.1. Then, $\mathcal{S}$ contains at least one $\mathcal{N}$-maximal element $(\bar{\sigma}, \bar{\theta}, \bar{g}, \bar{f}, \bar{u})$ whose domain is $D(\bar{c})$. We will next show that $\bar{c}=T$. To this aim, let us assume by contradiction that $\bar{c}<T$. We know that $\bar{u}(\bar{c}) \in D(\xi, \rho) \cap K$. 
Moreover, by using (12.1), (10.10), (12.2), we get

$$
\begin{aligned}
\|\bar{u}(\bar{c})-\xi\| & \\
& =\left\|S(\bar{c}) \xi+\int_{0}^{\bar{c}} S(\bar{c}-s) \bar{f}(s) d s+\int_{0}^{\bar{c}} S(\bar{\theta}(\bar{c}, s)) \bar{g}(s) d s-\xi\right\| \\
& \leq \sup _{t \in[0, \bar{c}]}\|S(t) \xi-\xi\|+\int_{0}^{\bar{c}}\|S(\bar{\theta}(\bar{c}, s)) \bar{g}(s)\| d s+\int_{0}^{\bar{c}}\|S(\bar{c}-s) \bar{f}(s)\| d s \\
& \leq \sup _{t \in[0, \bar{c}]}\|S(t) \xi-\xi\|+\bar{c} M e^{a T}(N+1)<\rho .
\end{aligned}
$$

Then, as $\bar{u}(\bar{c}) \in K$ and $F(\bar{u}(\bar{c})) \in \mathcal{Q T S}_{K}^{A}(\bar{u}(\bar{c}))$, there exist $\bar{\delta} \in(0, T-\bar{c}), \bar{\delta} \leq \varepsilon$, $\bar{f}_{0} \in L^{1}\left(\mathbb{R}_{+} ; F(\bar{u}(\bar{c}))\right)$ and $\bar{p} \in X,\|\bar{p}\| \leq \varepsilon$ such that

$$
S(\bar{\delta}) \bar{u}(\bar{c})+\int_{0}^{\bar{\delta}} S(\bar{\delta}-s) \bar{f}(s) d s+\bar{\delta} \bar{p} \in D(\xi, \rho) \cap K .
$$

Let us define the functions: $\widetilde{\sigma}:[0, \bar{c}+\bar{\delta}] \rightarrow[0, \bar{c}+\bar{\delta}], \widetilde{g}:[0, \bar{c}+\bar{\delta}] \rightarrow X$, $\widetilde{f}:[0, \bar{c}+\bar{\delta}] \rightarrow X$ and $\widetilde{\theta}:\{(t, s) ; 0 \leq s<t \leq \bar{c}+\bar{\delta}\} \rightarrow[0, \bar{c}+\bar{\delta}]$ by

$$
\begin{gathered}
\widetilde{\sigma}(t)=\left\{\begin{array}{cc}
\bar{\sigma}(t) & \text { for } t \in[0, \bar{c}] \\
\bar{c} & \text { for } t \in(\bar{c}, \bar{c}+\bar{\delta}],
\end{array}\right. \\
\widetilde{g}(t)=\left\{\begin{array}{cc}
\bar{g}(t) & \text { for } t \in[0, \bar{c}] \\
\bar{p} & \text { for } t \in(\bar{c}, \bar{c}+\bar{\delta}],
\end{array}\right. \\
\tilde{f}(t)=\left\{\begin{array}{cc}
\bar{f}(t) & \text { for } t \in[0, \bar{c}] \\
\bar{f}_{0}(t) & \text { for } t \in(\bar{c}, \bar{c}+\bar{\delta}],
\end{array}\right.
\end{gathered}
$$

and

$$
\tilde{\theta}(t, s)=\left\{\begin{array}{cl}
\bar{\theta}(t, s) & \text { for } 0 \leq s<t \leq \bar{c} \\
t-\bar{c}+\bar{\theta}(\bar{c}, s) & \text { for } 0 \leq s<\bar{c}<t \leq \bar{c}+\bar{\delta} \\
0 & \text { for } \bar{c} \leq s<t \leq \bar{c}+\bar{\delta}
\end{array}\right.
$$

Clearly, $\widetilde{\sigma}$ is nondecreasing, $\widetilde{g}$ and $\widetilde{f}$ are Bochner integrable on $[0, \bar{c}+\bar{\delta}], \widetilde{\theta}$ is measurable and they satisfy (i), (iii) and (iv).

Accordingly, we can define $\widetilde{u}:[0, \bar{c}+\bar{\delta}] \rightarrow X$ by

$$
\widetilde{u}(t)= \begin{cases}\bar{u}(t) & \text { if } t \in[0, \bar{c}] \\ S(t-\bar{c}) \bar{u}(\bar{c})+\int_{\bar{c}}^{t} S(t-s) \bar{f}_{0} d s+(t-\bar{c}) \bar{p} & \text { if } t \in[\bar{c}, \bar{c}+\bar{\delta}] .\end{cases}
$$

A standard calculation, involving the form of $\widetilde{\theta}$, shows that $\widetilde{u}$ satisfies (v). From (12.5), we get $\widetilde{u}(\bar{c}+\bar{\delta}) \in D(\xi, \rho) \cap K$ and thus (ii) is satisfied.

Following the same arguments as in the first part of the proof, we conclude that, diminishing $\bar{\delta}>0$ if necessary, we get also (vi). Thus $(\widetilde{\sigma}, \widetilde{\theta}, \widetilde{g}, \widetilde{f}, \widetilde{u})$ is an element of $\mathcal{S}$ which satisfies

$$
\mathcal{N}((\bar{\sigma}, \bar{\theta}, \bar{g}, \bar{f}, \bar{u}))<\mathcal{N}((\widetilde{\sigma}, \widetilde{\theta}, \widetilde{g}, \widetilde{f}, \widetilde{u}))
$$

although

$$
(\bar{\sigma}, \bar{\theta}, \bar{g}, \bar{f}, \bar{u}) \preceq(\widetilde{\sigma}, \widetilde{\theta}, \widetilde{g}, \widetilde{f}, \widetilde{u}) .
$$


This contradiction can be eliminated only if $\bar{c}=T$, and this completes the proof of Lemma 12.1 .

Remark 12.1. Under the general hypotheses of Lemma 12.1, for each $\gamma>0$, we can diminish both $\rho>0$ and $T>0$, such that $T<\gamma, \rho<\gamma$ and all the conditions (i) $\sim$ (vi) in Lemma 12.1 are satisfied.

\section{Proof of Theorem 11.1}

In this section we will prove that, in the hypotheses of Theorem 11.1, there exists at least one sequence of $\varepsilon_{n} \downarrow 0$ such that the corresponding sequence of $\varepsilon_{n}$ approximate mild solutions, $\left(\left(\sigma_{n}, \theta_{n}, g_{n}, f_{n}, u_{n}\right)\right)_{n}$, enjoys the property that $\left(u_{n}\right)_{n}$ is uniformly convergent on $[0, T]$ to some function $u:[0, T] \rightarrow K$ which is a mild solution of (10.1).

To this end, we need a necessary and sufficient condition in order that a given set of mild solutions be relatively compact in $C([\tau, T] ; X)$.

Definition 13.1. The operator $Q: X \times L^{1}(\tau, T ; X) \rightarrow C([\tau, T] ; X)$, defined by $Q(\xi, f)=u$, where $u$ is the unique mild solution of the problem

$$
\left\{\begin{array}{l}
u^{\prime}(t)=A u(t)+f(t) \\
u(0)=\xi
\end{array}\right.
$$

corresponding to $\xi \in X$ and $f \in L^{1}(\tau, T ; X)$, is called the mild solution operator attached to the problem (13.1).

Remark 13.1. The operator $Q$ is Lipschitz with constant $M e^{|a|(T-\tau)}$, where $M \geq 1$ and $a \in \mathbb{R}$ are given by (10.10), and therefore it maps bounded subsets in $X \times$ $L^{1}(\tau, T ; X)$ into bounded subsets in $C([\tau, T] ; X)$.

Theorem 13.1. Let $A: D(A) \subseteq X \rightarrow X$ be the generator of a $C_{0}$-semigroup $\{S(t): X \rightarrow X ; t \geq 0\}$, let $\mathcal{D}$ be a bounded subset in $X$, and $\mathcal{F}$ a uniformly integrable subset in $L^{1}(\tau, T ; X)$. Then $Q(\mathcal{D}, \mathcal{F})$ is relatively compact in $C([\theta, T] ; X)$ for each $\theta \in(\tau, T)$ if and only if there exists a dense subset $D$ in $[\tau, T]$ such that, for each $t \in D$, the $t$-section of $Q(\mathcal{D}, \mathcal{F})$, i.e., $Q(\mathcal{D}, \mathcal{F})(t)=\{Q(\xi, f)(t) ;(\xi, f) \in$ $\mathcal{D} \times \mathcal{F}\}$, is relatively compact in $X$. Moreover, if the latter condition is satisfied and $\tau \in D$, then $Q(\mathcal{D}, \mathcal{F})$ is relatively compact even in $C([\tau, T] ; X)$.

See Vrabie 24, Theorem 8.4.1, p. 194.

We are ready now to prove Theorem 11.1 .

Proof. Let $r>0, \rho \in(0, r]$ and $T>0$ as in Lemma 12.1. Since, by the hypotheses, $A+F$ is locally of compact type, diminishing $\rho \in(0, r]$ and $T>0$ if necessary, we can find a continuous function $\ell: \mathbb{R}_{+} \rightarrow \mathbb{R}_{+}$and a uniqueness function $\omega: \mathbb{R}_{+} \rightarrow \mathbb{R}_{+}$ such that $F(D(\xi, \rho) \cap K)$ is bounded and, for each $t \in[0, T]$ and $C \subseteq D(\xi, \rho) \cap K$, we have

$$
\beta(S(t) F(C)) \leq \ell(t) \omega(\beta(C)),
$$

and all the conclusions of Lemma 12.1 are satisfied. See Remark 12.1.

Let $\varepsilon_{n} \downarrow 0$ be a sequence in $(0,1)$ and let $\left(\left(\sigma_{n}, \theta_{n}, g_{n}, f_{n}, u_{n}\right)\right)_{n}$ be a sequence of $\varepsilon_{n}$-approximate mild solutions defined on $[0, T]$ whose existence is ensured by 
Lemma 12.1. From (v) 8 we have

$$
u_{n}(t)=S(t) \xi+\int_{0}^{t} S(t-s) f_{n}(s) d s+\int_{0}^{t} S\left(\theta_{n}(t, s)\right) g_{n}(s) d s
$$

for each $n \in \mathbb{N}$ and $t \in[0, T]$.

Throughout, $M \geq 1$ and $a \geq 0$ denote the constants satisfying (10.10).

We consider first the case when $X$ is separable. Let $t \in[0, T]$ and let us observe that, in view of (vi), for each $k \in \mathbb{N}$ and $s \in[0, t],\left\{u_{n}\left(\sigma_{n}(s)\right)-u_{n}(s) ; n \geq k\right\}$ is relatively compact and so $\beta\left(\left\{u_{n}\left(\sigma_{n}(s)\right)-u_{n}(s) ; n \geq k\right\}\right)=0$. Similarly, from (iii), it follows that $\beta\left(\left\{\int_{0}^{t} S(\theta(t, s)) g_{n}(s) d s ; n \geq k\right\}\right)=0$. From these remarks, Proposition 2.3, Lemma [2.3, (13.3), (10.10) and (13.2), we deduce

$$
\begin{aligned}
\beta & \left.\left\{u_{n}(t) ; n \geq k\right\}\right) \\
& \leq \beta\left(\left\{\int_{0}^{t} S(t-s) f_{n}(s) d s ; n \geq k\right\}\right)+\beta\left(\left\{\int_{0}^{t} S\left(\theta_{n}(t, s)\right) g_{n}(s) d s ; n \geq k\right\}\right) \\
& \leq \int_{0}^{t} \beta\left(\left\{S(t-s) f_{n}(s) ; n \geq k\right\}\right) d s \leq \int_{0}^{t} \ell(t-s) \omega\left(\beta\left(\left\{u_{n}\left(\sigma_{n}(s)\right) ; n \geq k\right\}\right)\right) d s \\
& \leq \int_{0}^{t} \sup _{\theta \in[0, T]} \ell(\theta) \omega\left(\beta\left(\left\{u_{n}(s) ; n \geq k\right\}+\left\{u_{n}\left(\sigma_{n}(s)\right)-u_{n}(s) ; n \geq k\right\}\right)\right) d s \\
& \leq \int_{0}^{t} \sup _{\theta \in[0, T]} \ell(\theta) \omega\left(\beta\left(\left\{u_{n}(s) ; n \geq k\right\}\right)\right) d s .
\end{aligned}
$$

Let us denote by $x_{k}(t)=\beta\left(\left\{u_{n}(t) ; n \geq k\right\}\right)$, for $k=1,2, \ldots$ and $t \in[0, T]$ and $\omega_{0}(r)=\sup _{\theta \in[0, T]} \ell(\theta) \omega(r)$, for $r \in \mathbb{R}_{+}$. The inequality above can be rewriten as

$$
x_{k}(t) \leq \int_{0}^{t} \omega_{0}\left(x_{k}(s)\right) d s
$$

for $k=1,2, \ldots$ and $t \in[0, T]$.

By Remark 2.2, $\omega_{0}$ is a uniqueness function. So, Lemma 2.5] shows that, diminishing $T>0$ if necessary, we may assume that $\lim _{k} x_{k}(t)=0$, which means that $\lim _{k} \beta\left(\left\{u_{n}(t) ; n \geq k\right\}\right)=0$ uniformly for $t \in[0, T]$. From Lemma 2.4 it follows that, for each $t \in[0, T],\left\{u_{n}(t) ; n=1,2, \ldots\right\}$ is relatively compact in $X$. At this point Theorem 13.1 comes into play and shows that there exists $u \in C([0, T] ; X)$ such that, on a subsequence at least, we have $\lim _{n} u_{n}(t)=u(t)$ uniformly for $t \in[0, T]$. In view of (ii) and (vi), we get $\lim _{n} u_{n}\left(\sigma_{n}(t)\right)=u(t)$ uniformly for $t \in[0, T]$, and, since $D(\xi, \rho) \cap K$ is closed, $u(t) \in D(\xi, \rho) \cap K$ for each $t \in[0, T]$.

Next, since $f_{n}(s) \in F\left(u_{n}\left(\sigma_{n}(s)\right)\right)$ for $n=1,2, \ldots$ and $s \in[0, T]$, and, by (12.1), $F(D(\xi, \rho) \cap K)$ is bounded, it follows that $\left\{f_{n} ; n=1,2, \ldots\right\}$ is uniformly integrable. Further, since $F$ is u.s.c. it is strongly-weakly u.s.c. too, and since $\left\{u_{n}\left(\sigma_{n}(s)\right) ; n=1,2, \ldots, s \in[0, T]\right\}$ is relatively compact, from Lemma 2.1 and Theorem 2.3, it follows that the set

$$
C=\overline{\mathrm{conv}} \bigcup_{n=1}^{\infty} \bigcup_{s \in[0, T]} F\left(u_{n}\left(\sigma_{n}(s)\right)\right)
$$

${ }^{8}$ Throughout this proof, the references to $(\mathrm{i}) \sim(\mathrm{vi})$ are to the corresponding items in Lemma 12.1 
is weakly compact. As $f_{n}(s) \in C$ for $n=1,2, \ldots$ and a.e. for $s \in[0, T]$, we are in the hypotheses of Corollary 2.1 which, along with Theorem 2.4, shows that we may assume without loss of generality that $\left(f_{n}\right)_{n}$ is weakly convergent in $L^{1}(0, T ; X)$ to some function $f$. Now Lemma 2.2 comes into play and shows that $f(s) \in$ $F(u(s))$ a.e. for $s \in[0, T]$. As the graph of the mild solution operator $Q$ : $L^{1}(0, T ; X) \rightarrow C([0, T] ; X)$, defined by $(Q g)(t)=S(t) \xi+\int_{0}^{t} S(t-s) g(s) d s$, for each $g \in L^{1}(0, T ; X)$, is weakly $\times$ strongly closed, being strongly $\times$ strongly closed, and convex, we may pass to the limit in (13.3), for $n \rightarrow \infty$. Taking into account of (iii), we obtain

$$
u(t)=S(t) \xi+\int_{0}^{t} S(t-s) f(s) d s,
$$

for each $t \in[0, T]$, and this concludes the proof in the case when $X$ is separable.

If $X$ is not separable, in view of Remark 2.1, it follows that there exists a separable and closed subspace, $Y$, of $X$ such that

$$
u_{n}(t), S(r) f_{n}(s), S\left(\theta_{n}(r, s)\right) g_{n}(s) \in Y
$$

for $n=1,2, \ldots$ and a.e. for $t, r, s \in[0, T]$. From Proposition 2.2 and the monotonicity of $\omega$, we deduce that

$$
\beta_{Y}(S(t) F(C)) \leq 2 \beta(S(t) F(C)) \leq 2 \ell(t) \omega(\beta(C)) \leq 2 \ell(t) \omega\left(\beta_{Y}(C)\right),
$$

for each $t>0$ and each set $C \subseteq D(\xi, \rho) \cap K \cap Y$. From now on, we have to repeat the routine in the separable case, by using the fact that the restriction of $\beta_{Y}$ (as defined in Proposition 2.2) to $\mathcal{B}(Y)$ is the Hausdorff measure of noncompactness on $Y$. This completes the proof.

\section{Proof of Theorem 11.3}

We indicate briefly how to show that, in the hypotheses of Theorem 11.3, there exists at least one sequence of $\varepsilon_{n} \downarrow 0$ such that the corresponding sequence of $\varepsilon_{n^{-}}$ approximate mild solutions, $\left(\left(\sigma_{n}, \theta_{n}, g_{n}, f_{n}, u_{n}\right)\right)_{n}$, enjoys the property that $\left(u_{n}\right)_{n}$ is uniformly convergent on $[0, T]$ to some function $u:[0, T] \rightarrow K$ which is a mild solution of (10.1).

Proof. Let $r>0, \rho \in(0, r]$ and $T>0$ as in Lemma12.1. Since $F$ is strongly-weakly u.s.c. and has weakly compact values, diminishing $\rho \in(0, r]$ and $T>0$ if necessary, we may assume that $F(K \cap D(\xi, \rho))$ is weakly relatively compact and, in addition, all the conclusions of Lemma 12.1 are satisfied. See Remark 12.1. From now on the proof follows, except for minor modifications, the very same arguments as those of the proof of Theorem 11.1, of course, with the help of (ii) in Remark 11.1

\section{Proof of Theorem 11.4}

We prove that there exists at least one sequence $\left(\varepsilon_{n}\right)_{n}$, with $\varepsilon_{n} \downarrow 0$, and such that the corresponding sequence $\left(\left(\sigma_{n}, \theta_{n}, g_{n}, f_{n}, u_{n}\right)\right)_{n}$, of $\varepsilon_{n}$-approximate mild solutions, enjoys the property that $\left(u_{n}\right)_{n}$ is uniformly convergent on $[0, T]$ to some function $u:[0, T] \rightarrow K$ which is a mild solution of (10.1). 
To this end we need a compactness lemma, which is an extension from continuous to Bochner integrable functions, of Vrabie [25, Lemma A.1.3, p. 295.

Lemma 15.1. Let $X$ be a Banach space, $K$ a compact subset in $X$ and let $\mathcal{F}$ be a family of Bochner integrable functions from $[\tau, T]$ to $K$. Then

$$
\left\{\int_{\tau}^{T} f(t) d t ; f \in \mathcal{F}\right\}
$$

is relatively compact in $X$.

Proof. Let $r>0, \rho \in(0, r]$ and $T>0$ as in Lemma 12.1. Since, by hypotheses, $K$ is locally compact and $F$ is strongly-weakly u.s.c. and has weakly compact values, diminishing $\rho \in(0, r]$ and $T>0$ if necessary, we may assume with no loss of generality that both $K \cap D(\xi, \rho)$ is compact and $F(K \cap D(\xi, \rho))$ is weakly relatively compact and, in addition, all the conclusions of Lemma 12.1 are satisfied. See Remark 12.1. Let $\varepsilon_{n} \downarrow 0$ be a sequence in $(0,1)$ and let $\left(\left(\sigma_{n}, \theta_{n}, g_{n}, f_{n}, u_{n}\right)\right)_{n}$ be a sequence of $\varepsilon_{n}$-approximate mild solutions defined on $[0, T]$ whose existence is ensured by Lemma 12.1. From (v) in Lemma 12.1. we have

$$
u_{n}(t)=S(t) \xi+\int_{0}^{t} S(t-s) f_{n}(s) d s+\int_{0}^{t} S\left(\theta_{n}(t, s)\right) g_{n}(s) d s
$$

for each $n \in \mathbb{N}$ and $t \in[0, T]$. From Lemma 15.1] it follows that, for each $t \in[0, T]$, the set $\left\{u_{n}(t)-\int_{0}^{t} S\left(\theta_{n}(t, s)\right) g_{n}(s) d s ; n=1,2 \ldots\right\}$ is relatively compact. Since $\lim _{n} g_{n}(t)=0$, uniformly for $t \in[0, T]$, an appeal to Theorem 13.1 shows that $\left\{u_{n} ; n=1,2, \ldots\right\}$ is relatively compact in $C([0, T] ; X)$. From now on the proof follows, except for minor modifications, the very same lines as those of the proof of Theorem 11.1.

\section{The Quasi-Autonomous Case}

Let $X$ be a Banach space, $A: D(A) \subseteq X \rightarrow X$ the infinitesimal generator of a $C_{0}$-semigroup, $\mathcal{C}$ a nonempty subset in $\mathbb{R} \times X, F: \mathcal{C} \leadsto X$ a multi-function and let us consider the Cauchy problem for the quasi-autonomous semilinear evolution inclusion

$$
\left\{\begin{array}{l}
u^{\prime}(t) \in A u(t)+F(t, u(t)) \\
u(\tau)=\xi
\end{array}\right.
$$

Let $X=\mathbb{R} \times X$ be endowed with the norm $\|(t, u)\| x=|t|+\|u\|$.

Definition 16.1. Let $A: D(A) \subseteq X \rightarrow X$ be the infinitesimal generator of a $C_{0}$-semigroup $\{S(t): X \rightarrow X ; t \geq 0\}$, and $F: \mathcal{C} \leadsto X$ a multi-function. We say that $A+F$ is locally of compact type with respect to the second argument if $F$ is u.s.c. and, for each $(\tau, \xi) \in \mathcal{C}$, there exist $\rho>0$, a continuous function $\ell: \mathbb{R}_{+} \rightarrow \mathbb{R}_{+}$ and a uniqueness function $\omega: \mathbb{R}_{+} \rightarrow \mathbb{R}_{+}$, such that

$$
\beta(S(t) F(C)) \leq \ell(t) \omega(\beta(C))
$$

for each $t>0$ and each $C \subseteq D_{X}((\tau, \xi), \rho) \cap \mathcal{C}$.

Definition 16.2. By a mild solution of the quasi-autonomous multi-valued semilinear Cauchy problem (16.1), we mean a continuous function $u:[\tau, T] \rightarrow X$, with 
$(t, u(t)) \in \mathcal{C}$ for each $t \in[\tau, T]$, and for which there exists $f \in L^{1}(\tau, T ; X)$ such that $f(s) \in F(s, u(s))$ a.e. for $s \in[\tau, T]$ and

$$
u(t)=S(t-\tau) \xi+\int_{\tau}^{t} S(t-s) f(s) d s,
$$

for each $t \in[\tau, T]$.

Definition 16.3. The set $\mathcal{C} \subseteq \mathbb{R} \times X$ is mild viable with respect to $A+F$ if for each $(\tau, \xi) \in \mathcal{C}$, there exists $T \in \mathbb{R}, T>\tau$ such that the Cauchy problem (16.1) has at least one mild solution $u:[\tau, T] \rightarrow X$.

Remark 16.1. The quasi-autonomous Cauchy problem (16.1) can be equivalently rewritten as an autonomous one in the space $\mathcal{X}$, by setting $\mathcal{A}=(0, A), z(s)=$ $(t(s+\tau), u(s+\tau)), \mathcal{F}(z)=(1, F(z)) \sqrt{9}$ and $\zeta=(\tau, \xi)$. Indeed, with the notation above, we have

$$
\left\{\begin{array}{l}
z^{\prime}(s) \in \mathcal{A} z(s)+\mathcal{F}(z(s)) \\
z(0)=\zeta
\end{array}\right.
$$

It readily follows that $\mathcal{A}$ generates a $C_{0}$-semigroup $\{\mathcal{S}(t): \mathcal{X} \rightarrow \mathcal{X} ; t \geq 0\}$ on $X$, where $\mathcal{S}(t)=(1, S(t))$ for each $t \geq 0,\{S(t): X \rightarrow X ; t \geq 0\}$ being the $C_{0}$-semigroup generated by $A$ on $X$.

Remark 16.2. One may easily see that $\mathcal{C}$ is mild viable with respect to $A+F$ in the sense of Definition 16.3 if and only if $\mathcal{C}$ is mild viable with respect to $\mathcal{A}+\mathcal{F}$ in the sense of Definition 10.2 .

Theorem 16.1. Let $A: D(A) \subseteq X \rightarrow X$ be the infinitesimal generator of a $C_{0^{-}}$ semigroup $\{S(t): X \rightarrow X ; t \geq 0\}$. Let $\mathcal{C}$ be a nonempty subset in $\mathbb{R} \times X$ and $F: \mathcal{C} \leadsto X$ a nonempty, quasi-weakly compact and convex valued, u.s.c. multifunction. If $\mathcal{C}$ is mild viable with respect to $A+F$, then, for each $(\tau, \xi) \in \mathcal{C}$, we have

$$
(1, F(\tau, \xi)) \in \mathcal{Q T S}_{\mathcal{C}}^{\mathcal{A}}(\tau, \xi) .
$$

Proof. From Remark 16.2 we know that $\mathcal{C}$ is mild viable with respect to $\mathcal{A}+\mathcal{F}$ in the sense of Definition 10.2 and thus, by Theorem 10.1, we conclude that, under the hypotheses of Theorem 16.1, for each $z \in \mathcal{C}, z=(\tau, \xi)$, we have

$$
\mathcal{F}(z) \in \mathcal{Q T}_{\mathcal{C}}^{\mathcal{A}}(z),
$$

a relation which is equivalent to (16.3).

Likewise, in the autonomous case, we have:

Theorem 16.2. Let $X$ be a Banach space, and $A: D(A) \subseteq X \rightarrow X$ be the infinitesimal generator of a $C_{0}$-semigroup $\{S(t): X \rightarrow X ; t \geq 0\}$. Let $\mathcal{C}$ be a nonempty subset in $\mathbb{R} \times X$ and $F: \mathcal{C} \leadsto X$ a given multi-function. If $\mathcal{C}$ is viable with respect to $A+F$, then the tangency condition

$$
(1, F(\tau, \xi)) \cap \mathcal{T}_{\mathcal{C}}^{\mathcal{A}}(\tau, \xi) \neq \emptyset
$$

is satisfied at each upper semi-continuity point, $(\tau, \xi) \in \mathcal{C}$, of $F$, at which $F$ is convex and compact.

Proof. Use Remark 16.2 and Theorem 10.3

\footnotetext{
${ }^{9}$ Here $(1, F(z))=\{(1, \eta) ; \eta \in F(z)\}$.
} 
From Theorem 16.1 and Remark 10.5 we deduce :

Theorem 16.3. Let $X$ be reflexive and let $A: D(A) \subseteq X \rightarrow X$ be the infinitesimal generator of a $C_{0}$-semigroup $\{S(t): X \rightarrow X ; t \geq 0\}$. Let $\mathcal{C}$ be a nonempty subset in $\mathbb{R} \times X$ and $F: \mathcal{C} \leadsto X$ a nonempty, closed and convex valued, u.s.c. multi-function. If $\mathcal{C}$ is mild viable with respect to $A+F$, then, for each $(\tau, \xi) \in \mathcal{C}$, the tangency condition (16.3) is satisfied.

We can now pass to the main sufficient conditions concerning the viability of a set $\mathcal{C}$ with respect to $A+F$.

Theorem 16.4. Let $X$ be a Banach space, $A: D(A) \subseteq X \rightarrow X$ the infinitesimal generator of a $C_{0}$-semigroup $\{S(t): X \rightarrow X ; t \geq 0\}$, $\mathcal{C}$ a nonempty and locally closed subset in $\mathbb{R} \times X$ and $F: \mathcal{C} \leadsto X$ a nonempty, weakly compact, closed and convex valued multi-function such that $A+F$ is locally of compact type with respect to the second argument. If, for each $(\tau, \xi) \in \mathcal{C}$, the tangency condition (16.3) is satisfied, then $\mathcal{C}$ is mild viable with respect to $A+F$.

Proof. We have

$$
\beta(\mathcal{S}(t) \mathcal{F}(C)) \leq \beta(S(t) F(C)) \leq \ell(t) \beta(C),
$$

for each bounded set $C$ in $X$ and each $t>0$. Thus, if $A+F$ is locally of compact type with respect to the second argument, then $\mathcal{A}+\mathcal{F}$ is locally of compact type (in the space $X$ ) in the sense of Definition [1.1. So the conclusion follows from Remark 16.2 and Theorem 11.1.

Theorem 16.5. Let $X$ be a Banach space, $A: D(A) \subseteq X \rightarrow X$ be the infinitesimal generator of a compact $C_{0}$-semigroup $\{S(t): X \rightarrow X ; t \geq 0\}$, C a nonempty and locally closed subset in $\mathbb{R} \times X$ and $F: \mathcal{C} \leadsto X$ a nonempty, weakly compact and convex valued multi-function which is strongly-weakly u.s.c. If, for each $(\tau, \xi) \in \mathcal{C}$, the tangency condition (16.3) is satisfied, then $\mathrm{C}$ is mild viable with respect to $A+F$.

Proof. Just use Remark 16.2 and Theorem 11.3.

The next result is a simple corollary of Theorem 16.2 Remark 5.2 and Theorem 16.4.

Theorem 16.6. Let $X$ be a Banach space, $A: D(A) \subseteq X \rightarrow X$ the infinitesimal generator of a $C_{0}$-semigroup $\{S(t): X \rightarrow X ; t \geq 0\}$, $\mathrm{C}$ a nonempty and locally closed subset in $\mathbb{R} \times X$ and $F: \mathcal{C} \leadsto X$ a nonempty, closed and convex valued, locally $\beta$-compact multi-function. Then $\mathcal{C}$ is mild viable with respect to $A+F$ if and only if, for each $(\tau, \xi) \in \mathcal{C}$, the tangency condition (16.4) is satisfied.

\section{Global mild solutions}

Let $A: D(A) \subseteq X \rightarrow X$ be the infinitesimal generator of a $C_{0}$-semigroup, let $\mathcal{C} \subseteq \mathbb{R} \times X$ be nonempty and let $F: \mathcal{C} \leadsto X$ be a given multi-function. In this section we will state some results concerning the existence of noncontinuable, or even global mild solutions to the Cauchy problem

$$
\left\{\begin{array}{l}
u^{\prime}(t) \in A u(t)+F(t, u(t)) \\
u(\tau)=\xi
\end{array}\right.
$$

A mild solution $u:[\tau, T) \rightarrow X$ of (17.1) is called noncontinuable if there is no other mild solution $v:[\tau, \widetilde{T}) \rightarrow X$ of the same equation, with $T<\widetilde{T}$ and satisfying 
$u(t)=v(t)$ for all $t \in[\tau, T)$. The mild solution $u$ is called global if $T=T_{\mathcal{C}}$, where $T_{\mathrm{e}}$ is given by (9.2).

The next theorem follows from the Brezis-Browder Theorem 2.1] and its proof is very similar to the one of Theorem 9.1

Theorem 17.1. Let $X$ be a Banach space, $A: D(A) \subseteq X \rightarrow X$ be the infinitesimal generator of a $C_{0}$-semigroup, $\mathcal{C}$ a nonempty subset in $\mathbb{R} \times X$ and $F: \mathcal{C} \leadsto X$ a given multi-function. The following conditions are equivalent:

(i) $\mathcal{C}$ is viable with respect to $A+F$;

(ii) for each $(\tau, \xi) \in \mathcal{C}$ there exists at least one noncontinuable mild solution $u:[\tau, T) \rightarrow X$ of $(17.1)$.

We conclude with a result concerning the existence of global solutions.

Theorem 17.2. Let $X$ be a Banach space, let $A: D(A) \subseteq X \rightarrow X$ be the infinitesimal generator of a $C_{0}$-semigroup of contractions and let $\mathcal{C}$ be an $X$-closed subset in $\mathbb{R} \times X$. Let $F: \mathcal{C} \leadsto X$ be a multi-function such that $\mathcal{C}$ is mild viable with respect to $A+F$. If $F$ is positively sublinear 10 and maps bounded subsets in $\mathrm{C}$ into bounded subsets in $X$, then each mild solution of (17.1) can be continued up to a global one, i.e., defined on $\left[\tau, T_{\mathfrak{C}}\right)$.

Proof. Since $\mathcal{C}$ is mild viable with respect to $A+F$, for each $(\tau, \xi) \in \mathcal{C}$, there exists at least one noncontinuable mild solution $u:[\tau, T) \rightarrow X$ to (17.1). We will show that $T=T_{\mathrm{C}}$. To this aim, let us assume the contrary, i.e., that $T<T_{\mathcal{C}}$. From Benilan's inequality (see for instance Vrabie 23]) we deduce

$$
\|u(t)\| \leq\|\xi\|+\int_{E_{t}}[u(s), f(s)]_{+} d s+\int_{H_{t} \backslash G_{t}}[u(s), f(s)]_{+} d s
$$

for each $t \in[\tau, T)$, where $f$ is the function given by Definition 10.1, and

$$
\begin{aligned}
& E_{t}=\left\{s \in[\tau, t] ;[u(s), f(s)]_{+}>0 \text { and }\|u(s)\|>c(s)\right\}, \\
& G_{t}=\left\{s \in[\tau, t] ;[u(s), f(s)]_{+} \leq 0\right\}, \\
& H_{t}=\{s \in[\tau, t] ;\|u(s)\| \leq c(s)\} .
\end{aligned}
$$

Here $a, b, c: \mathbb{R} \rightarrow \mathbb{R}_{+}$are the continuous functions in Definition 9.1, As $[u, v]_{+} \leq$ $\|v\|$ for each $u, v \in X$, we get

$$
\|u(t)\| \leq\|\xi\|+\int_{E_{t}}[a(s)\|u(s)\|+b(s)] d s+\int_{H_{t}}\|f(s)\| d s
$$

for each $t \in[\tau, T)$. But $F$ maps bounded subsets in $\mathcal{C}$ into bounded subsets in $X$ and therefore there exists $m>0$ such that $\|f(s)\| \leq m$ a.e. for $s \in H_{t}$ and for all $t \in[\tau, T)$. Hence

$$
\|u(t)\| \leq\|\xi\|+T m+\int_{\tau}^{T} b(s) d s+\int_{\tau}^{t} a(s)\|u(s)\| d s,
$$

for each $t \in[\tau, T)$. By Gronwall's Lemma, $u$ is bounded on $[\tau, T)$.

Using once again the fact that $F$ maps bounded subsets in $\mathcal{C}$ into bounded subsets in $X$, we deduce that $f$ is bounded on $[\tau, T)$ and therefore, there exists $\lim _{t \uparrow T} u(t)=u^{*}$. Since $\mathcal{C}$ is $X$-closed, it follows that $\left(T, u^{*}\right) \in \mathcal{C}$. From this observation, recalling that $\mathcal{C}$ is $C^{0}$-viable with respect to $A+F$ and $T<+\infty$, we conclude that $u$ can be continued to the right of $T$. But this is absurd, because

\footnotetext{
${ }^{10}$ See Definition 9.1
} 
$u$ is noncontinuable. This contradiction can be eliminated only if $T=T_{\mathcal{C}}$, as claimed.

Remark 17.1. Theorem 17.2 remains true in the more general case when $A: D(A) \subseteq$ $X \rightarrow X$ is the infinitesimal generator of a $C_{0}$-semigroup of type $(1, a)$, with $a>0$. In order to prove Theorem 17.2 in this general case we have to consider $A-a I$ instead of $A$ and to repeat the above proof using the equality $[x, a x+y]_{+}=[x, y]_{+}+a\|x\|$.

Remark 17.2. Recall that the $C_{0}$-semigroup is of type $(1, a)$ if

$$
\|S(t) x\| \leq e^{a t}\|x\|
$$

for every $x \in X$ and $t \geq 0$. Recall further that for every $C_{0}$-semigroup $\{S(t): X \rightarrow$ $X ; t \geq 0\}$ there exists a norm on $X$, equivalent to the initial one, such that, with respect to this new norm, the $C_{0}$-semigroup is of type $(1, a)$.

\section{A Controllability PRoBlem}

Let $X$ be a Banach space, $A: D(A) \subseteq X \rightarrow X$ the infinitesimal generator of a $C_{0}$-semigroup $\{S(t): X \rightarrow X ; t \geq 0\}, g: X \rightarrow X$ a given function, $\xi \in X$, and $c(\cdot)$ a measurable control taking values in $D(0,1)$. Here, the problem we consider is how to find a control $c(\cdot)$ in order to reach the origin starting from the initial point $\xi$ in some time $T$, by mild solutions of the state equation

$$
\left\{\begin{array}{l}
u^{\prime}(t)=A u(t)+g(u(t))+c(t) \\
u(0)=\xi
\end{array}\right.
$$

Let us consider $G: X \leadsto X$, defined by $G(x)=g(x)+D(0,1)$. We can rewrite the above problem as follows. For a given $\xi \in X$, find $T>0$ and a mild solution of the multi-valued semilinear Cauchy problem

$$
\left\{\begin{array}{l}
u^{\prime}(t) \in A u(t)+G(u(t)) \\
u(0)=\xi
\end{array}\right.
$$

that satisfies $u(T)=0$.

The main result of this section is given by the following theorem.

Theorem 18.1. Let $g: X \rightarrow X$ be a continuous function such that for some $L>0$ we have

$$
\|g(x)\| \leq L\|x\|,
$$

for every $x \in X$. Assume that $X$ is reflexive, and the semigroup $\{S(t): X \rightarrow$ $X ; t \geq 0\}$ is compact and satisfies the condition (17.2) for some $a \in \mathbb{R}$. Then, for every $\xi \in X$ with $\xi \neq 0$ there exists a mild solution $u:[0, \infty) \rightarrow X$ of (18.2) which satisfies

$$
\|u(t)\| \leq\|\xi\|-t+(L+a) \int_{0}^{t}\|u(s)\| d s
$$

for every $t \geq 0$ for which $u(t) \neq 0$.

Corollary 18.1. Under the hypothesis of Theorem 18.1, the following properties hold. 
(i) In case $L+a \leq 0$, for any $\xi \in X, \xi \neq 0$, there exist a control $c(\cdot)$ and $a$ mild solution of (18.1) that reaches the origin of $X$ in some time $T \leq\|\xi\|$ and satisfies

$$
\|u(t)\| \leq\|\xi\|-t
$$

for any $0 \leq t \leq T$.

(ii) In case $L+a>0$, for every $\xi \in X$ satisfying $0<\|\xi\|<1 /(L+a)$, there exist a control $c(\cdot)$ and a mild solution of (18.1) that reaches the origin of $X$ in some time

$$
T \leq \frac{1}{L+a} \log \frac{1}{1-(L+a)\|\xi\|}
$$

and satisfies

$$
\|u(t)\| \leq e^{(L+a) t}\left(\|\xi\|-\frac{1}{L+a}\right)+\frac{1}{L+a}
$$

for any $0 \leq t \leq T$.

We notice that, in view of Remark 17.2, the condition (17.2) can always be satisfied if we replace the initial norm with an equivalent one. We begin with the proof of Corollary 18.1 .

Proof. In case (i), since $L+a \leq 0$, by (18.4) we deduce that there exists a mild solution $u:[0, \infty) \rightarrow X$ of (18.2) which satisfies the inequality $\|u(t)\| \leq\|\xi\|-t$ for every $t \geq 0$ for which $u(t) \neq 0$. This implies that there exists $T>0$ with $T \leq\|\xi\|$ such that $u(T)=0$. By Definition 10.1, there exists $c \in L^{1}(0, T ; X)$ such that $c(s) \in D(0,1)$ a.e. for $s \in[0, T]$ and $u$ is a mild solution of (18.1). This completes the proof of (i). To prove (ii) we proceed similarly, by observing that (18.6) comes from (18.4), via the Gronwall Lemma, with $x(\cdot)=\|u(\cdot)\|-\frac{1}{L+a}$.

We now proceed with the proof of Theorem 18.1.

Proof. We consider the space $\mathbb{R} \times X$, the operator $\mathcal{A}=(0, A)$ that generates the $C_{0}$-semigroup $(1, S(t))$ on $\mathbb{R} \times X$, the locally closed set

$$
K=\left\{(\lambda, x) \in \mathbb{R}_{+} \times X \backslash\{0\} ;\|x\| \leq \lambda\right\},
$$

and the multi-function $F: \mathbb{R} \times X \leadsto \mathbb{R} \times X$ defined by

$$
F(t, x)=((L+a)\|x\|-1, g(x)+D(0,1)),
$$

for every $(t, x) \in \mathbb{R} \times X$. We show that

$$
((L+a)\|\xi\|-1, g(\xi)+D(0,1)) \in 2 \mathcal{T S}_{K}^{\mathcal{A}}(\lambda, \xi),
$$

for every $(\lambda, \xi) \in K$. In view of Remark 10.1, to prove this it suffices to check that, for each $\xi \in X, \xi \neq 0$, there exist $\left(h_{n}\right)_{n},\left(\theta_{n}\right)_{n}$ both in $\mathbb{R}$, and $\left(g_{n}\right)_{n} \in \mathcal{G}(\xi) 11$ with $h_{n} \downarrow 0$ and $\lim _{n} \theta_{n}=0$ and such that

$$
\begin{aligned}
& \left\|S\left(h_{n}\right) \xi+\int_{0}^{h_{n}} S\left(h_{n}-s\right) g_{n}(\xi) d s\right\| \\
& \leq\|\xi\|+h_{n}((L+a)\|\xi\|-1)+h_{n} \theta_{n} .
\end{aligned}
$$

\footnotetext{
${ }^{11}$ We recall that $\mathcal{G}(\xi)=\left\{g \in L^{1}\left(\mathbb{R}_{+} ; X\right) ; g(s) \in G(\xi)\right.$ a.e. for $\left.s \in \mathbb{R}_{+}\right\}$. See also Defini-
} tion 10.3 
We will consider $a \neq 0$, the case $a=0$ following by simpler arguments. Namely, let us first observe that

$$
\begin{gathered}
\left\|S(h) \xi+\int_{0}^{h} S(h-s) g(\xi) d s-\int_{0}^{h} S(h-s) S(s) e^{-a s} \frac{\xi}{\|\xi\|} d s\right\| \\
=\left\|S(h) \xi+\int_{0}^{h} S(h-s) g(\xi) d s+\frac{1}{a\|\xi\|}\left(e^{-a h}-1\right) S(h) \xi\right\| \\
\leq\|S(h) \xi\|\left(1+\frac{1}{a\|\xi\|}\left(e^{-a h}-1\right)\right)+\left\|\int_{0}^{h} S(h-s) g(\xi) d s\right\| \\
\leq e^{a h}\|\xi\|-\frac{1}{a}\left(e^{a h}-1\right)+\left\|\int_{0}^{h} S(h-s) g(\xi) d s\right\|,
\end{gathered}
$$

for $h$ sufficiently small. Further,

$$
\lim _{h \downarrow 0}\left(\frac{e^{a h}-1}{h}\|\xi\|-\frac{e^{a h}-1}{a h}+\left\|\frac{1}{h} \int_{0}^{h} S(h-s) g(\xi) d s\right\|\right) \leq(L+a)\|\xi\|-1 .
$$

From these inequalities, it is easy to see that for any arbitrary sequence $\left(h_{n}\right)_{n}$, with $h_{n} \downarrow 0$, there exist $\left(\theta_{n}\right)_{n}$ in $\mathbb{R}, \theta_{n} \downarrow 0$, and $\left(g_{n}\right)_{n}$, defined by

$$
g_{n}(s)=g(\xi)-S(s) e^{-a s} \frac{\xi}{\|\xi\|} \in g(\xi)+D(0,1),
$$

for $n=1,2, \ldots$ and a.e. for $s \geq 0$, and such that $\left(h_{n}\right)_{n},\left(\theta_{n}\right)_{n}$ and $\left(g_{n}\right)_{n}$ satisfy the conditions in Remark10.1, Thus, we get (18.7). Moreover, from Theorems 11.1 and 17.1. we deduce that for each $\xi \in X, \xi \neq 0$, there exist $T>0$ and a noncontinuable mild solution $(z, u):[0, T) \rightarrow \mathbb{R} \times X$ of the Cauchy problem

$$
\left\{\begin{array}{l}
z^{\prime}(t)=(L+a)\|u(t)\|-1 \\
u^{\prime}(t) \in A u(t)+G(u(t)) \\
z(0)=\|\xi\| \text { and } u(0)=\xi
\end{array}\right.
$$

which satisfies $(z(t), u(t)) \in K$ for every $t \in[0, T)$. This means that (18.4) is satisfied for every $t \in[0, T)$.

Now, let us observe that $u$, as a solution of (18.2), can be continued to $\mathbb{R}_{+}$simply because $G$ has sublinear growth. So, $u(T)$ exists, even though the solution $(z, u)$ of (18.8) is defined merely on $[0, T)$. Clearly $u(T)$ must be 0 since otherwise, $(z, u)$ can be continued to the right of $T$, thereby contradicting the fact that $(z, u)$ is noncontinuable. This completes the proof.

For a very recent result obtained via completely different arguments but similar to Theorem 18.1 (allowing $A$ to generate an arbitrary $C_{0}$-semigroup and $g$ to be Lipschitz), see Cârjă [5].

\section{REFERENCES}

[1] J. P. Aubin and A. Cellina, Differential inclusions, Springer-Verlag, Berlin-Heidelberg-New York-Tokyo, 1984. MR755330 (85j:49010)

[2] W. Bebernes and I. D. Schuur, The Ważewski topological method for contingent equations, Ann. Mat. Pura Appl., 87(1970), 271-278.

[3] H. Brezis and F. E. Browder, A general principle on ordered sets in nonlinear functional analysis, Adv. in Mathematics, 21(1976), 355-364. MR0425688 (54:13641) 
[4] H. Bouligand, Sur les surfaces dépourvues de points hyperlimités, Ann. Soc. Polon. Math., $\mathbf{9}(1930), 32-41$.

[5] O. Cârjă, On the minimal time function and the minimum energy problem: a nonlinear case, Systems Control Lett., 55 (2006), 543-548. MR2225363 (2007b:93018)

[6] O. Cârjă and M. D. P. Monteiro Marques, Weak tangency, weak invariance and Carathéodory mappings, J. Dynam. Control Systems, 8(2002), 445-461. MR1931893 (2003k:34033)

[7] O. Cârjă and C. Ursescu, The characteristics method for a first order partial differential equation, An. Ştiin. Univ. Al. I. Cuza Iaşi, Secţ. I a Mat., 39(1993), 367-396. MR1328937 (96h:35252)

[8] O. Cârjă and I. I. Vrabie, Some new viability results for semilinear differential inclusions, NoDEA Nonlinear Differential Equations Appl., 4(1997), 401-424. MR1458535 (98h:34029)

[9] F. H. Clarke, Yu. S. Ledyaev and M. L. Radulescu, Approximate invariance and differential inclusions in Hilbert spaces, J. Dynam. Control Systems, 3 (1997), pp. 493-518. MR1481624 (98k:49011)

[10] K. Deimling, Nonlinear functional analysis, Springer-Verlag, Berlin Heidelberg New York Tokyo, 1985. MR787404 (86j:47001)

[11] J. Diestel, Remarks on weak compactness in $L_{1}(\mu ; X)$, Glasg. Math. J., 18(1977), 87-01.

[12] J. Diestel and J. J. Uhl, Jr., Vector measures, Mathematical Surveys, 15, Amer. Math. Soc., Providence, RI, 1977. MR0453964 (56:12216)

[13] N. Dunford and J. T. Schwartz, Linear operators Part I: General theory, Interscience Publishers, Inc., New York, 1958. MR1009162 (90g:47001a)

[14] R. E. Edwards, Functional analysis theory and applications, Holt, Rinehart and Winston, New York Chicago San Francisco Toronto London, 1965. MR0221256 (36:4308)

[15] S. Gautier, Equations differentielles multivoques sur un fermé, Publications de l'Université de Pau, (1973).

[16] E. Hille and R. S. Phillips, Functional analysis and semi-groups, Amer. Math. Soc. Colloquium Publications, 31, Fourth Printing of Revised Edition, 1981. MR0089373 (19:664d)

[17] H. Mönch, Boundary value problems for nonlinear ordinary differential equations of second order in Banach spaces, Nonlinear Anal. T.M.A., 4(1980), 985-999. MR586861 (82c:34075)

[18] M. Nagumo, Über die Lage der Integralkurven gewöhnlicher Differentialgleichungen, Proc. Phys.-Math. Soc. Japan, 24(1942), 551-559. MR0015180 (7:381e)

[19] N. H. Pavel and I. I. Vrabie, Equations d'évolution multivoques dans des espaces de Banach, C. R. Acad. Sci. Paris Sér. A-B, 287(1978), A315-A317. MR0513204(58:23829)

[20] N. H. Pavel and I. I. Vrabie, Semilinear evolution equations with multivalued right-hand side in Banach spaces, An. Ştiinţ. Univ. Al. I. Cuza Iaşi Secţ. I a Mat., 25(1979), 137-157. MR.553132 (81a:47060)

[21] F. Severi, Su alcune questioni di topologia infinitesimale, Annales Soc. Polonaise, 9(1931), 97-108.

[22] S. Z. Shi, Viability theorems for a class of differential operator inclusions, J. Differential Equations, 79(1989), 232-257. MR1000688 (90e:34025)

[23] I. I. Vrabie, Compactness methods for nonlinear evolutions, Second Edition, Pitman Monographs and Surveys in Pure and Applied Mathematics 75, Longman, 1995. MR.1375237 (96k:47116)

[24] I. I. Vrabie, $C_{0}$-semigroups and applications, North-Holland Publishing Co. Amsterdam, 2003. MR.1972224 (2004c:47088)

[25] I. I. Vrabie, Differential equations. An introduction to basic concepts, results and applications, World Scientific Publishing Co., Inc., River Edge, NJ, 2004. MR2092912 (2005d:34001)

Faculty of Mathematics, "Al. I. CuzA" University, IaŞi 700506, Romania - and "Octav Mayer" Mathematics Institute, Romanian Academy, Iaşi 700506, Romania

E-mail address: ocarja@uaic.ro

Faculty of Mathematics, "Al. I. CuzA" University Iaşi 700506, Romania

E-mail address: necula@uaic.ro

Faculty of Mathematics, "Al. I. CuzA" University, Iași 700506, Romania - AND "Octav MayeR" Mathematics Institute, Romanian Academy, Iaşi 700506, Romania

E-mail address: ivrabie@uaic.ro 